\title{
k-space optical microscopy of nanoparticle arrays: Opportunities and artifacts
}

Jean-François Bryche, Grégory Barbillon, Bernard Bartenlian, Gérald Dujardin, Elizabeth Boer-Duchemin, and Eric Le Moal

Citation: Journal of Applied Physics 124, 043102 (2018); doi: 10.1063/1.5029976

View online: https://doi.org/10.1063/1.5029976

View Table of Contents: http://aip.scitation.org/toc/jap/124/4

Published by the American Institute of Physics

AIP $\left.\right|_{\text {Applied Physics }} ^{\text {Journal of }}$ SPECIAL TOPICS

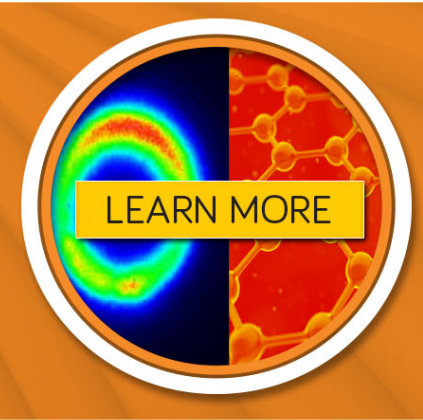




\title{
k-space optical microscopy of nanoparticle arrays: Opportunities and artifacts
}

\author{
Jean-François Bryche, ${ }^{1,2}$ Grégory Barbillon, ${ }^{1,3}$ Bernard Bartenlian, ${ }^{1}$ Gérald Dujardin, ${ }^{4}$ \\ Elizabeth Boer-Duchemin, ${ }^{4}$ and Eric Le Moal ${ }^{4, a)}$ \\ ${ }^{1}$ Centre de Nanosciences et de Nanotechnologies, CNRS, Univ. Paris-Sud, Université Paris-Saclay, \\ C2N - Orsay, 91405 Orsay Cedex, France \\ ${ }^{2}$ Laboratoire Charles Fabry, CNRS, Institut d'Optique Graduate School, Univ. Paris-Sud, Université \\ Paris-Saclay, 2 Avenue Augustin Fresnel, 91127 Palaiseau Cedex, France \\ ${ }^{3}$ EPF-Ecole d'Ingénieurs, 3 bis rue Lakanal, 92330 Sceaux, France \\ ${ }^{4}$ Institut des Sciences Moléculaires d'Orsay (ISMO), CNRS, Univ. Paris-Sud, Université Paris-Saclay, \\ 91405 Orsay Cedex, France
}

(Received 17 March 2018; accepted 2 July 2018; published online 24 July 2018)

\begin{abstract}
We report on the performance and inherent artifacts of $k$-space optical microscopy for the study of periodic arrays of nanoparticles under the various illumination configurations available on an inverted optical microscope. We focus on the origin of these artifacts and the ways to overcome or even benefit from them. In particular, a recently reported artifact, called the "condenser effect," is demonstrated here in a new way. The consequences of this artifact (which is due to spurious reflections in the objective) on Fourier-space imaging and spectroscopic measurements are analyzed in detail. The advantages of using $k$-space optical microscopy to determine the optical band structure of plasmonic arrays and to perform surface plasmon resonance experiments are demonstrated. Potential applications of $k$-space imaging for the accurate lateral and axial positioning of the sample in optical microscopy are investigated. Published by AIP Publishing.

https://doi.org/10.1063/1.5029976
\end{abstract}

\section{INTRODUCTION}

Most often in optical microscopy, only an intensity image is recorded and the phase information is ignored. As a result, the wavevector ( $k$-vector) content of the light emitted from the sample cannot be obtained simply from a Fourier transform of the real-space image. Nevertheless, the $k$-vector information may be retrieved from the intensity distribution of the collected light in the back focal plane of the microscope objective, i.e., from the Fourier-space image. ${ }^{1,2}$ This technique, referred to as $k$-space or Fourier-space optical microscopy, is increasingly used by researchers, especially for the study of periodic nanoparticle arrays. ${ }^{3-8}$

Periodic arrays of metallic nanoparticles on transparent substrates and their complementary structures, i.e., periodic nanohole arrays etched in metallic layers, have been widely studied for their unique optical properties. ${ }^{9}$ These properties include the existence of spectrally sharp collective optical resonances, ${ }^{10-17}$ the ability to control spontaneous emission, ${ }^{18-21}$ the ability to disperse ${ }^{22}$ and focus light, ${ }^{23,24}$ the ability to support lasing, ${ }^{25,26}$ and the extraordinary transmission of light through nanohole arrays. ${ }^{27,28}$ Numerous applications of nanoparticles and nanohole arrays as chemical and biological sensors have been described in the recent literature. $^{29-35}$ In addition, periodic arrays of metallic nanoparticles on metallic films have attracted special interest for the engineering of the dispersion of surface plasmon polaritons (SPPs), ${ }^{36-38}$ for the design of micro-optics for SPP launching and decoupling, ${ }^{39}$ waveguiding, ${ }^{40}$ multiplexing, ${ }^{41}$

a)eric.le-moal@u-psud.fr and steering, ${ }^{42-44}$ and for lasing, ${ }^{45}$ biosensing, ${ }^{46,47}$ and photovoltaic $^{48}$ applications.

In several of the most recent publications cited above, ${ }^{18,20,21,25,26,42-44} k$-space optical microscopy is key in the elucidation of the investigated physical mechanisms. Recently, it has also been reported that $k$-space optical microscopy may be used to take advantage of a previously disregarded artifact from microscope objectives, called the "condenser effect," in order to resolve the geometry of periodic nanoparticle arrays beyond the optical diffraction limit. ${ }^{49-52}$ However, only an indirect demonstration for the origin of the "condenser effect" is available in the literature, ${ }^{50}$ and the consequences of this artifact on spectroscopic measurements have been unaddressed until now.

In this paper, we report on some of the most interesting possibilities and artifacts of $k$-space optical microscopy for the study of periodic nanoparticle arrays. Three different illumination configurations available in a liquid-immersionobjective-equipped inverted optical microscope are used. First, we describe in Sec. III A how the Fourier-space image of a periodic array is formed in the microscope, and we guide the reader in the interpretation of this image. We provide in Sec. III B a new and more direct demonstration of the "condenser effect," and we discuss the ways to overcome or even benefit from this artifact. We quantitatively analyze in Sec. III C the contribution of this artifact to the total detected light and describe in Sec. IIID the consequences of the "condenser effect" on spectroscopic measurements. Next, in Sec. IIIE, we focus on the advantages of using $k$-space optical microscopy for the study of the SPP band structure of periodically nanostructured metallic layers. Finally, we 
describe in Sec. III F potential applications of $k$-space optical microscopy and periodic nanostructure arrays for the accurate lateral and axial positioning of the sample with respect to the illumination focus.

\section{SAMPLES AND EXPERIMENTAL SETUP}

\section{A. Sample fabrication}

Figure 1 shows scanning electron microscopy (SEM) images of the samples used in this study. The samples are fabricated using an e-beam lithography technique. ${ }^{53,54}$ They consist of periodic arrays of cylindrical gold nanodots, whose height and diameter are $30 \mathrm{~nm}$ and $200 \mathrm{~nm}$, respectively. The choice of these geometrical parameters is motivated by previous studies on similar nanodot arrays used as sensors or for fundamental studies of their plasmonic properties. ${ }^{16,17,53-55}$ The nanodot arrays are lithographed on a $170 \mu \mathrm{m}$-thick glass coverslip that is either bare or coated with a $85 \mathrm{~nm}$-thick indium tin oxide (ITO) layer or with a $30 \mathrm{~nm}$ or $50 \mathrm{~nm}$-thick gold film. The ITO films are purchased from SOLEMS, Palaiseau, France. The gold films are grown in vacuum at a base pressure of $5 \times 10^{-7}$ mbar using an electron beam evaporator [PLASSYS, used at the micronanotechnology platform of the Center for Nanosciences and Nanotechnologies (C2N-CTU) in Orsay, France]. Prior to gold deposition, a $2 \mathrm{~nm}$-thick titanium layer is deposited in the same vacuum chamber in order to improve adhesion to the substrate.

For the e-beam lithography process, an $80 \mathrm{~nm}$-thick layer of polymethylmethacrylate A2 (PMMA A2) resist is first spin-coated on the substrate and baked. By design, an
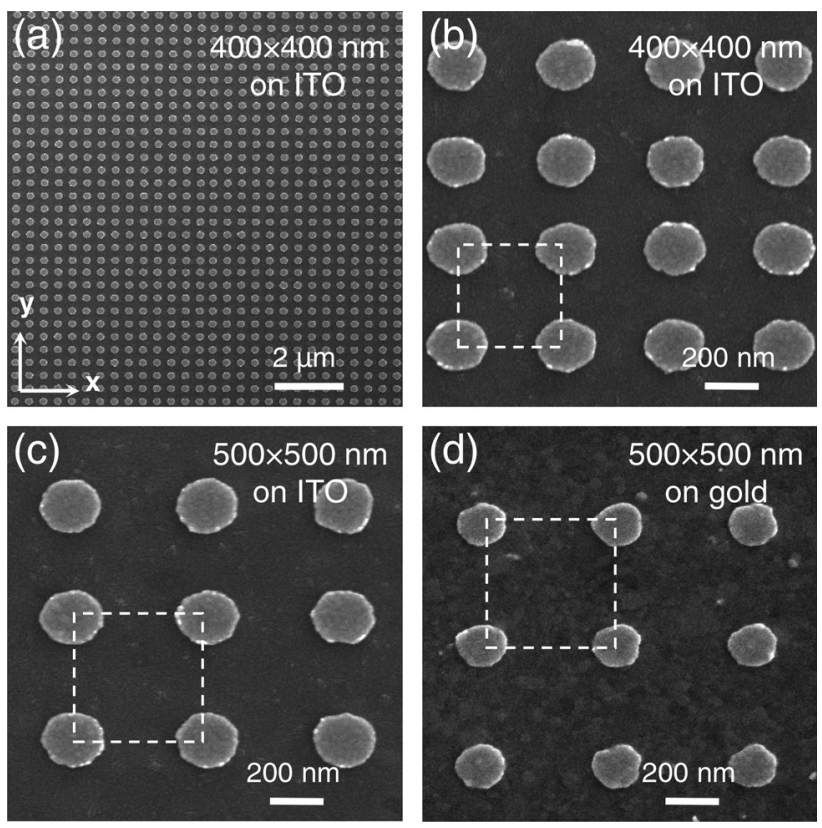

FIG. 1. Scanning electron microscopy (SEM) images of the samples used in this study. The samples consist of periodic arrays of gold nanodots, $30 \mathrm{~nm}$ in height and $200 \mathrm{~nm}$ in diameter, which are nanolithographed on an $85 \mathrm{~nm}$ thick ITO layer [(a)-(c)] or a $50 \mathrm{~nm}$-thick gold film on a glass coverslip (d). The array has square symmetry with periods $400 \mathrm{~nm}$ [(a) and (b)] and $500 \mathrm{~nm}[(\mathrm{c})$ and (d)]. The SEM image magnification is $6500 \times$ (a) and $50000 \times[(\mathrm{b})-(\mathrm{d})]$. array occupies a $250 \times 250 \mu \mathrm{m}^{2}$ area on the substrate. Ebeam lithography is then performed at a current of $2 \mathrm{nA}$. A development step in a 1:3 methylisobutylketone/isopropanol (MIBK/IPA) solution is used to reveal the written pattern. Next, a 30-nm thick gold layer is evaporated on the sample. Finally, the nanostructures are obtained after a liftoff process in acetone. The same process as described above is performed on the ITO- and gold-coated substrates. For the bare glass substrates, an extra step is added: a thin layer of the conducting polymer Espacer $300 \mathrm{Z}$ is spin-coated on top of the resist before e-beam lithography in order to avoid charging. ${ }^{56,57}$ This conducting layer is removed using deionized water before the development of the resist. All nanostructure dimensions are confirmed using scanning electron microscopy and atomic force microscopy measurements. Film thicknesses are verified using ellipsometry and X-ray measurements.

\section{B. Optical microscopy}

All experiments are carried out using an inverted optical microscope (Nikon Instruments, Eclipse Ti-U) equipped with an oil-immersion, high numerical aperture (NA) objective lens. In particular, a Nikon CFI Apochromat $100 \times 1.49$ NA TIRF objective is used. (TIRF stands for total-internalreflection fluorescence microscopy.) The microscope is coupled to a cooled CCD camera (Andor, IKON-M) and an imaging spectrometer (Horiba Jobin Yvon, iHR320 spectrometer and Synapse CCD detector). We use a set of removable achromatic doublet lenses that are arranged in a $4 f$ geometry (as in a telescope or a Fourier filtering system) in such a way that the setup can be switched from real-space to the Fourier-space imaging mode by simply removing two lenses and adding another without changing the detector position. Thus, an optical image with spatial coordinates (real-space) or angular coordinates (Fourier-space) is projected directly onto the detector or, using a removable mirror, onto the entrance slit of the imaging spectrometer. The $k$-scale of the Fourier-space images is calibrated using the SPP leakage radiation angle on a 50-nm thick gold film on glass as a reference, following the method described in Ref. 58.

Figure 2 shows schematically the different illumination configurations used in this study. In the first two configurations [Figs. 2(a) and 2(b)], the initial illumination is through the transparent substrate, and the objective lens is used for both the illumination of the sample and for the collection of the light that is reflected or back scattered (episcopic configuration). The initial light beam may be (a) focused on the sample or (b) collimated with normal $\left(\theta_{\text {inc }}=0^{\circ}\right)$ or tilted $\left(\theta_{\text {inc }}>0^{\circ}\right)$ incidence. Here, we use a CW linearly polarized He-Ne laser (vacuum wavelength $\lambda_{0}=632.8 \mathrm{~nm}$ ). These configurations are described in more detail below.

In the configuration shown in Fig. 2(a), the objective lens is used to focus the laser beam onto the substrate-sample interface and to collect the reflected light. The laser beam is expanded using a telescope to completely fill the rear pupil of the microscope objective (the width of the expanded beam is larger than the inner diameter of the pupil). Thus, the 
Reflected light (episcopic) illumination

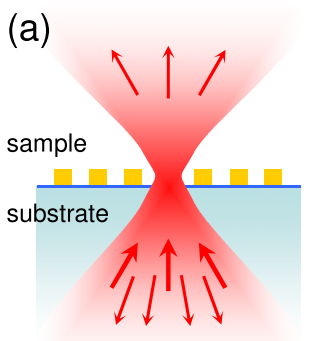

focused laser beam (b)

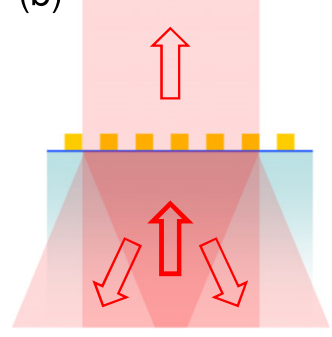

collimated laser beam normal incidence
Transmitted light (diascopic) illumination

(c)

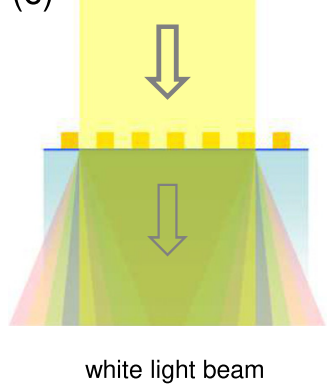

FIG. 2. Schematics of the illumination configurations used in this study. In (a) and (b), the sample is illuminated through the substrate with (a) a focused or (b) a collimated laser beam, and the reflected and back-scattered light is collected in reflection using the same objective lens. In (c), the sample is illuminated from above with a collimated white light beam, and the transmitted and scattered light is collected in transmission using the objective lens.

reflectivity of the substrate-sample interface may be measured for all incident angles within the acceptance cone of the objective lens in a single Fourier-space image. Moreover, since the initial beam is linearly polarized, the focused incident light is $s$-polarized in one plane and $p$-polarized in a perpendicular plane. Thus, the reflectivity for both $s$ - and $p$-polarized incidence may be measured simultaneously.

The configuration shown in Fig. 2(b) is similar to a standard light diffraction setup, where a quasi-plane wave is back scattered from a $2 \mathrm{D}$ periodic grating in directions that are determined by the period of the grating and the wavelength of the incident light. In Fourier space, a diffraction pattern is observed, where bright spots on a dark background correspond to the diffraction orders of the periodic sample. In the following, we refer to these spots using $(i, j)$ Miller indices. Moreover, by varying the angle of incidence $\left(\theta_{i n c}>0^{\circ}\right)$, this configuration may also be used as a Surface Plasmon Resonance (SPR) system ${ }^{59}$ as shown in Sec. III E (additional experimental details can be found in the supplementary material).

In addition, in a final configuration, the incident light originates from above the sample and the transmitted light is collected using the microscope objective (diascopic configuration). This is illustrated in Fig. 2(c). The incident beam is from a collimated white light source (from a Köhler illumination system included in the microscope apparatus) and arrives from above the sample at normal incidence $\left(\theta_{\text {inc }}=0^{\circ}\right)$. An iris diaphragm is used to control the diameter of the incident beam. Bandpass (BP) interferometric filters are used to select narrow spectral bands from the broad emission spectrum of the lamp. A linear polarizer is set in front of the light source to control the orientation of the incident electric field with respect to the main axes of the array $\left(\mathbf{E}_{\text {inc }} \| \mathbf{x}\right.$ or $\left.\mathbf{E}_{\text {inc }} \| \mathbf{y}\right)$. Here, we use a white light source in order to investigate the spectral dependence of the optical response of the sample.

\section{RESULTS AND DISCUSSION}

\section{A. Interpreting the Fourier-space image}

In this section, we describe how the Fourier-space image is formed in the optical microscope and how it may be analyzed in order to retrieve geometrical information about a periodic sample. Here, we focus on the possibilities offered by $k$-space optical microscopy when it is used to study nanodot arrays on transparent or semi-transparent substrates which do not support surface waves. Further possibilities specific to the study of nanodot arrays on metal-coated substrates (which support SPPs) are described in Sec. III E. Here, we first use the reflected light illumination configuration with a focused laser beam which is introduced in Fig. 2(a). One motivation for the use of this configuration is that the "condenser effect" (addressed in Sec. III B) does not occur, and thus, the description of image formation in $k$-space optical microscopy is simplified.

Figure 3(a) shows a real-space image where both the gold nanodots of a $500 \times 500 \mathrm{~nm}$ periodic array and the laser spot may be seen due to additional wide-field, white light illumination of the sample. The laser beam is focused using the high-NA objective lens to a spot whose dimensions are almost at the diffraction limit. The width at half intensity maximum of the laser spot $(<250 \mathrm{~nm})$ is thus smaller than the unit cell of the array $(a=500 \mathrm{~nm})$. When keeping only the laser illumination and saturating the contrast of the image as has been done in Fig. 3(b), one can see, however, that light is scattered from gold nanodots that are several micrometers away from the focal point. Indeed, the nanodots at the focal point can scatter the incident light in all in-plane directions. This light can then be scattered out-of-plane by nanodots further away. Thus, tens to hundreds of nanodots actually contribute to the total scattered light. As a result, even though the incident beam is tightly focused, the scattered light is expected to be sensitive to the geometry of the nanodot array, at least to within an area of a few tens of $\mu \mathrm{m}^{2}$ of the incident beam. The effect of the array periodicity on the scattered light is confirmed in the Fourier-space observations discussed below.

Figures 3(c) and 3(d) show the Fourier-space images obtained when the laser spot lies within the nanodot array and when it is far away from it, respectively. When the laser beam does not interact with the gold nanostructures [Fig. 3(d)], only the back reflection of the incident light from the substrate-air interface is seen in the Fourier-space image. As expected, internal reflection is weak at subcritical angles $\left(\frac{k_{\|}}{k_{0}}=n \sin \theta<1\right)$ and total at supercritical angles $\left(\frac{k_{\|}}{k_{0}}>1\right)$. 

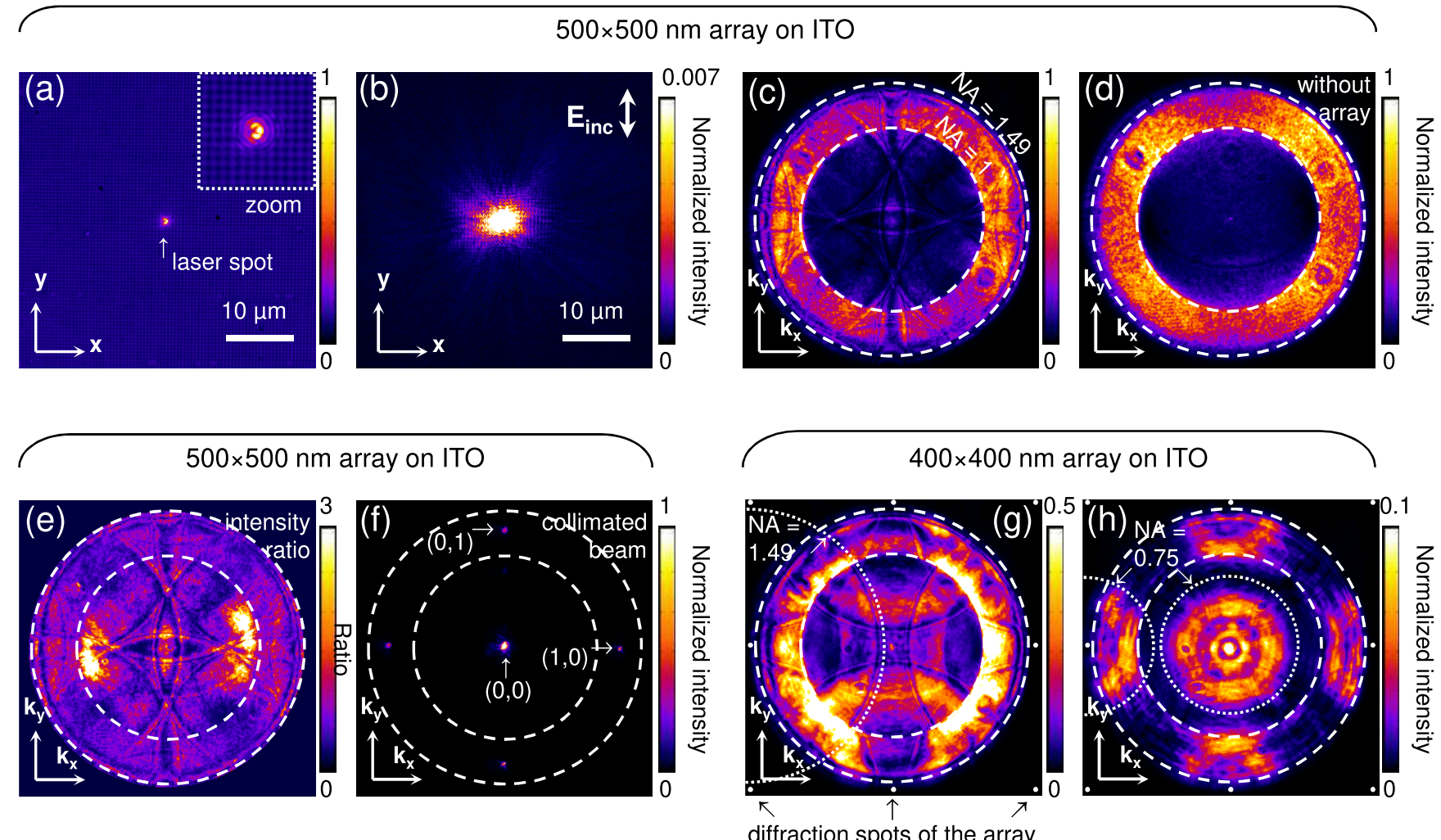

FIG. 3. Real- and Fourier-space optical microscopy images of periodic gold nanodot arrays on ITO-coated glass, with periodicities of $500 \times 500 \mathrm{~nm}$ in (a) to (f) and $400 \times 400 \mathrm{~nm}$ in $(\mathrm{g})$ and (h). Intensity images are in false color (see the color scale on the right). All images are obtained in a reflected-light illumination configuration using a focused laser beam, as shown in Fig. 2(a), except in (f), where a collimated laser beam in normal incidence is used, as in Fig. 2(b). In (a), transmitted white light illumination is added to see the array which extends over the whole field of view. In (b), the intensity scale is saturated so that the lateral extension of the excitation may be seen. In [(c)-(f)], the two dashed circles indicate the maximal collection angle of the objective lens (NA $=n_{\text {glass }} \sin \theta_{\max }$ $=1.49, \theta_{\max } \approx 79^{\circ}$ ) and the critical angle at the air/glass interface (NA $=n_{\text {glass }} \sin \theta_{c}=1, \theta_{c} \approx 41^{\circ}$ ), respectively. In (d), the measurement is carried out in an area where there are no nanostructures present. In (e), the image is obtained by dividing the image shown in (c) by that shown in (d). In (g) and (h), white dots indicate the position of the diffraction spots. In (g), the laser beam completely fills the rear pupil of the objective, whereas in (h), the beam width is reduced so as to fill only the central area of the pupil. In the latter case, the sample is illuminated with a light cone whose angular aperture is limited to $\theta_{\text {inc }}=30^{\circ}$ (equivalent to $\mathrm{NA}=0.75)$. In all images, except in (e), the intensity scale is normalized with respect to the intensity maximum.

Thus, except for artificial spots due to dust on the CCD detector or bubbles in the oil droplet on the objective lens, the Fourier-space image of the structureless area is featureless. Conversely, when the laser spot is focused on the array, as is the case in Fig. 3(c), the result is a tangle of dark and bright narrow rings.

Figure $3(\mathrm{~g})$ shows an image obtained under similar conditions but on a different (this time $400 \times 400 \mathrm{~nm}$ ) periodic array of gold nanodots. Dark and bright narrow rings of the same radius as those in Fig. 3(c), the $500 \times 500 \mathrm{~nm}$ array image, are again seen; however, in the two images [Figs. $3(\mathrm{c})$ and $3(\mathrm{~g})]$, the rings are centered on different points in Fourier space. These rings do not result from the so-called "condenser effect" discussed in Refs. 49-52, which is an artifact that can only occur under diascopic (i.e., transmitted light) illumination. The origin of the observed rings may be understood from Fig. 3(h), where an iris diaphragm is used to reduce the diameter of the laser beam entering the rear pupil of the objective (thus limiting the "illumination NA"). When the experiment is repeated with the lower-NA illumination, we see that the "illumination pattern" (within the circle of NA $=0.75$ ) is replicated and shifted to specific points of Fourier space which correspond to the diffraction orders of the periodic array.
The same conclusion may be drawn for the $500 \times 500 \mathrm{~nm}$ array from Figs. 3(e) and 3(f). Figure 3(f) is obtained when the nanodot array is illuminated with a collimated laser beam in normal incidence and shows the position of the diffraction orders of the nanodot array. In Fig. 3(e), the Fourier-space image of the nanodot array is normalized by that of a structureless area in order to increase the visibility of the interesting features. Looking closely, we can now see that the ring centers coincide with the observed diffraction spots. Moreover, the observed rings, which have radii which are slightly smaller than the circle of $\mathrm{NA}=1.49$, also appear centered on the $(0,0)$ spot. Such rings do not appear in Fig. 3(d) for the structureless area. They are not a simple intensity feature of the reflected light image but must be a phase feature of the reflection pattern. The dark and bright narrow rings seen in Figs. 3(c) and $3(\mathrm{~g})$ result from the interference between the pattern centered on $(0,0)$ and those shifted to $(i, j) \neq(0,0)$ or, in other words, from the interference between the back-reflected light and the light diffracted by the array.

Why do we see these rings at a radius that is slightly smaller than the circle of NA $=1.49$ ? This is due to a wellknown optical phenomenon whose effect increases with the incidence angle. Namely, light is known to undergo a phase shift upon total internal reflection at a dielectric-dielectric 
interface. ${ }^{60,61}$ This phase shift monotonously increases from 0 at the critical angle to $\pi \mathrm{rad}$ at $\theta_{i n c}=90^{\circ}$. This is actually the same phenomenon that is responsible for the GoosHänchen effect, where a finite sized beam undergoes a lateral shift upon total internal reflection. The effect observed in our case is a pure phase effect and would thus not be detected in intensity images if the reflected light did not interfere with the light diffracted from the periodic nanodot array. Because of the Abbe sine condition (which is fulfilled in the type of objective used), $k_{\|} / k_{0}=\sin \theta_{i n c}$, and the angle of incidence $\theta_{\text {inc }}$ and the phase shift increase more rapidly as the position in Fourier space moves to the rear pupil edge. As a result, the interference fringes are most easily observed close to the circle of radius $\mathrm{NA}=1.49$. A second effect, occurring inside the microscope objective, may further shift the phase of the reflected and diffracted light at supercritical angles, especially when using high-NA objectives that are designed to compensate for image aberrations due to phase delay at high emission angles; a description of this additional effect can be found in the supplementary material.

As discussed above, the images shown in Figs. 3(c) and $3(\mathrm{f})$ are the diffraction patterns of a focused laser beam and a collimated laser beam on a periodic nanostructure array, as measured using the illumination configurations introduced in Figs. 2(a) and 2(b), respectively. Both images may be used to characterize the orientation, symmetry, and periodicity of the nanostructure array. These geometrical parameters are obtained from the coordinates of the diffraction spots in Fourier space. However, the configuration used in Fig. 3(c) has a major advantage over the one used in Fig. 3(f), which is the possibility to resolve these geometrical parameters beyond the diffraction limit. Indeed, in Fig. 3(c), the coordinates of the diffraction spots may be determined even when they exceed the maximum $k$ values accessible using the optical microscope (note that $\left(\left|\mathbf{k}_{\mathbf{x}}\right|^{2}+\left|\mathbf{k}_{\mathbf{y}}\right|^{2}\right)_{\max }=k_{0}^{2} \mathrm{NA}^{2}$, where $k_{0}=2 \pi / \lambda_{0}$ ). The incomplete circles in the Fourier image are centered on the diffraction spots. Thus, the position of these diffraction spots in Fourier space may be obtained by fitting an entire circle to the observed segments even when the diffraction spots are not visible [see an example in Figs. 3(g) and 3(h)]. Thus, in principle, the spatial periods $P$ of the array may be measured for $P>P_{\min }$ with $P_{\min }=\lambda_{0} /(2 \mathrm{NA})$ (e.g., $P_{\min }=213 \mathrm{~nm}$ for $\lambda_{0}=632.8 \mathrm{~nm}$ and $\mathrm{NA}=1.49$ ). In contrast, no such circles occur using the other configuration; only the diffraction spots are expected, and they may only be detected if they lie inside the disk of radius $k_{0} \mathrm{NA}$. As a result, the evaluation of the spatial period is limited to values of $P$ larger than $\lambda_{0} /(\mathrm{NA})$.

In addition, the illumination configuration used in Fig. 3 (c) has potential applications in the field of positioning metrology. This is true since the intensity distribution in the Fourier-space image is highly sensitive to the relative position of the laser spot with respect to the nanodot array. A detailed description of this effect and its use in the accurate lateral and axial positioning of the sample is given in Sec. III F.

\section{B. The "condenser effect"}

Here, we address the issue of the "condenser effect," an artifact that occurs under diascopic illumination [Fig. 2(c)] and which was previously reported in Refs. 49-52. First of all, we present results that confirm the occurrence of the "condenser effect" when studying a periodic array of nanostructures on a transparent substrate, i.e., the appearance of artifactual rings in $k$-space microscopy images due to spurious reflections in the objective. Interestingly, this effect may also be used to characterize the spatial periodicity of the array beyond the transmission limit of the microscope objective, in a way that is similar to what was shown in Sec. III A for episcopic illumination. A model for the origin of the "condenser effect" has been proposed in Ref. 50 on the basis of experiments requiring a modified setup with two objective lenses. Below, we introduce a more direct and simpler way to reveal the origin of this artifact using a single standard microscope objective. Our results unambiguously verify the following hypothesis: incident light that is internally reflected in the front objective lens (i.e., in the first of the series of lenses that make up the collection objective) is then scattered from the circular edge of the lens mount back to the sample. Finally, we explain why the "condenser effect" generally does not occur when the periodic array of nanostructures lies on a metal-coated substrate (e.g., a thin gold film on a glass slide).

Figure 4 shows the Fourier-space images that are obtained when a periodic array of gold nanodots on an ITOcoated glass substrate is illuminated by white light in transmission using the configuration described in Fig. 2(c). For the first row of images, the width of the incident light beam exceeds the apparent diameter of the front objective lens (i.e., the diameter of the aperture in the metallic housing into which the lens is sealed). For the second row of images, the width of the incident light beam is smaller than the apparent diameter of the objective. In both cases, the first image in the series is recorded without spectral filtering; thus, the pattern in Fourier space is averaged over the broad spectrum of the white light source. Interferometric bandpass (BP) filters are used in the next two images in order to select narrow spectral bands (full-width-at-half-maximum, $13 \mathrm{~nm}$ ) centered at $\lambda_{0}$ $=650$ and $740 \mathrm{~nm}$, respectively. The last of the four images is obtained on an unstructured area of the sample, far from any gold nanodot, and is used as a reference.

As is seen by comparing Figs. 4(a) and 4(e), the diffraction pattern of the nanostructure array appears in the Fourierspace image regardless of whether the incident beam diameter $\varnothing_{\text {beam }}$ exceeds the front lens diameter $\emptyset_{\text {lens }}$ or not. However, in the former case $\left(\varnothing_{\text {beam }}>\emptyset_{\text {lens }}\right)$, the Fourierspace image exhibits an additional feature which resembles a four-leaf clover in Fig. 4(a). It is only when the front edge of the collection lens is illuminated that the artifact occurs. Spectrally filtering the image reveals that the clover-shaped feature is the result of the sum of sharp circular rings centered on the diffraction spots of the array. The radius of these circles is equal to or slightly larger than the radius corresponding to the maximum collection angle (i.e., the circle of radius $k_{0} \mathrm{NA}$ ). These observations indicate that the rings in Figs. 4(b) and 4(c) are due to the illumination of the array from the substrate side with light that has scattered from the edge of the metallic casing that holds the front lens. This artifact was named the "condenser effect" $"$ by analogy to 

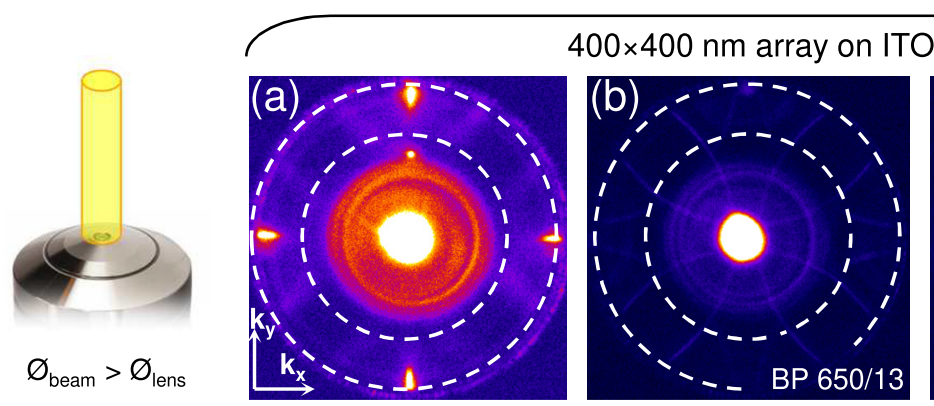

without array
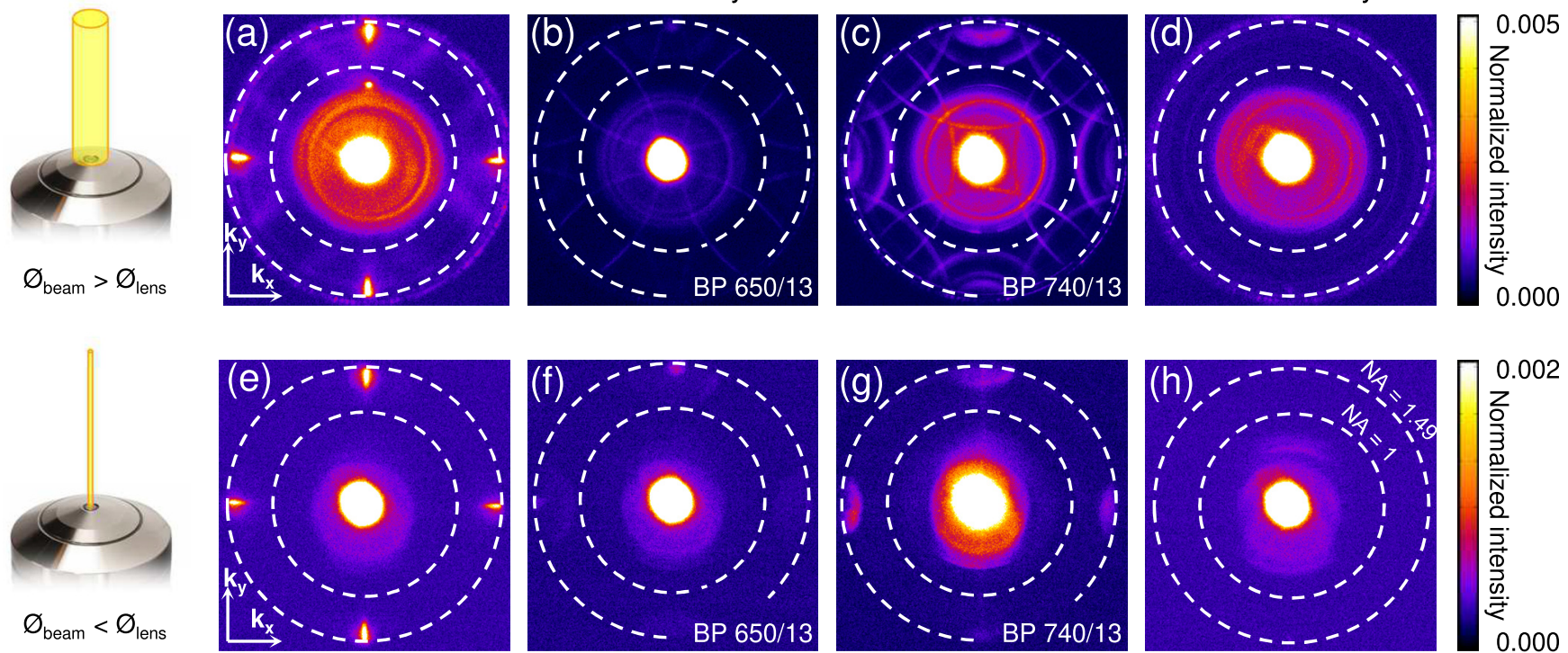

FIG. 4. Fourier-space optical microscopy images of a $400 \times 400 \mathrm{~nm}$ periodic gold nanodot array on ITO-coated glass. All images are obtained in a transmitted light illumination configuration using a collimated white light beam with normal incidence, as shown in Fig. 2(c). In [(a)-(d)], the diameter of the incident light beam is larger than the front aperture of the microscope objective, whereas in [(e)-(h)], the beam diameter is smaller than the front aperture. Bandpass filters are used in [(b) and (c)] and [(f) and (g)] (see the wavelength and bandwidth in the bottom right-hand corner of the image). In (d) and (h), measurements are carried out on an area without nanostructures. In each image, the intensity is divided by its maximal value, which corresponds to the intensity of the transmitted light at $\theta=0$ (i.e., the central spot). In all images, the intensity scale is normalized with respect to the $(0,0)$ spot intensity.

the optical condensers used in microscopy since the resulting scattered radiation which illuminates the sample has a particular form. Below, we use a new method to elucidate the origin of the "condenser effect" which differs from the previously reported, more demanding, methods ${ }^{50,51}$ in its simplicity.

Figure 5 shows the results of an experiment similar to that described in Fig. 4 except that the incident light beam is partially blocked above the sample using a piece of opaque paper. Thus, the aperture of the metallic casing that holds the objective front lens may be completely (first column in Fig. 5 ) or partially illuminated (second and third columns). In the second column of Fig. 5, more than half the area of the front lens is illuminated, which includes the field of view of the microscope objective (a $0.2 \mathrm{~mm}$-wide area centered on the optical axis). In this case, the diffraction spots of the array remain unchanged in the image, but some of the incomplete circles are absent, as compared to the images shown in the first column. We infer that the light scattered from the edge of the lens toward the sample yields a conical illumination pattern, whose angular distribution corresponds to the sharp circular rings that appear in Figs. 5(f) and 5(i). The blocking part of the incident beam results in missing rings, as shown in Figs. 5(g) and 5(j). In the third column of Fig. 5, less than half of the area of the front lens is illuminated. In particular, the field of view of the microscope objective is blocked and no light directly from the source arrives in this region. As a result, the only light that illuminates the observed area on the sample is the light that has scattered from the unblocked side of the lens edge. No diffraction spots are seen in the Fourierspace images in this case since no light illuminates the array in normal incidence. (The array occupies a $250 \times 250 \mu \mathrm{m}^{2}$ area on the sample, which almost fits the objective's field of view, and is much smaller than the top side of the front lens, which has a diameter of $3 \mathrm{~mm}$.) The incomplete circles remain however, thus confirming that their origin is the incident light scattered from the lens casing.

Figure 6 is used to explain the observed effect in detail. A axial cut of the microscope objective is shown where the hemispherical front lens and part of its cylindrical mount are seen. In principle, a light ray (I) impinging on the edge of the lens mount may scatter in all directions in the surrounding transparent media, i.e., in the oil and glass. Within a very narrow solid angle (a few angular degrees in the incident plane), the scattered light from the aperture edge reaches the field of view of the microscope objective which is more than ten times smaller than the front lens diameter [see Fig. 6(a)]; the resulting illumination of the sample is similar to that obtained using an annular condenser. The internal reflection of the light from the top interface of the sample is not expected to be collected since the angle of incidence is equal to or slightly larger than the angular aperture of the lens; however, the periodic array on the sample can diffract the light in directions that are within the collection cone. The involved combination of wavevectors is described in Fig. 6(c). Upon diffraction at the array, the in-plane wavevector component $\mathbf{k}_{\|, \mathbf{S}}$ of the scattered light from the aperture is modified by the addition of a Bragg vector $\mathbf{K}_{\overline{\mathbf{1}} \mathbf{0}}$; the resulting wavevector lies within the collection cone, with $\left|\mathbf{k}_{\|, \mathbf{D}}\right|=\left|\mathbf{k}_{\|, \mathbf{S}}+\mathbf{K}_{\overline{\mathbf{1}, \mathbf{0}}}\right|<k_{0} \mathrm{NA}$.

As shown in Fig. 6(b), light impinging on the edge of the aperture can also scatter into the glass lens $\left(S_{1}\right)$ and undergo a number of total internal reflections before scattering out from the opposite side of the circular aperture. In a similar but mirrored situation as compared to the above, the scattered light $\left(S_{2}\right)$ from the edge can be diffracted by the 

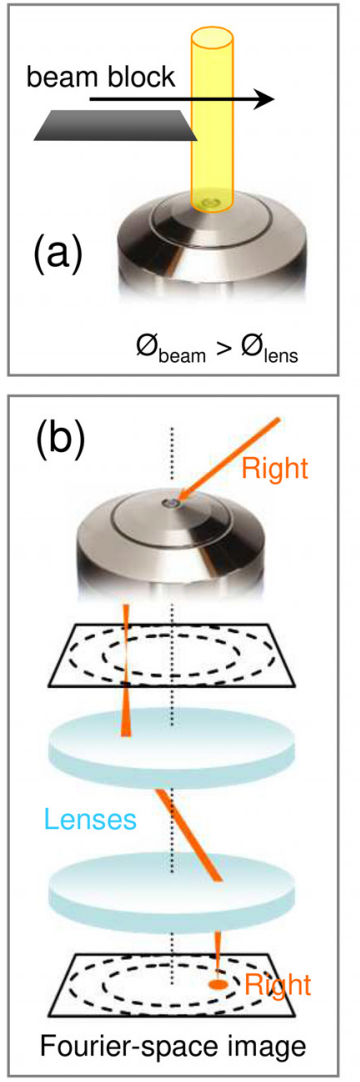
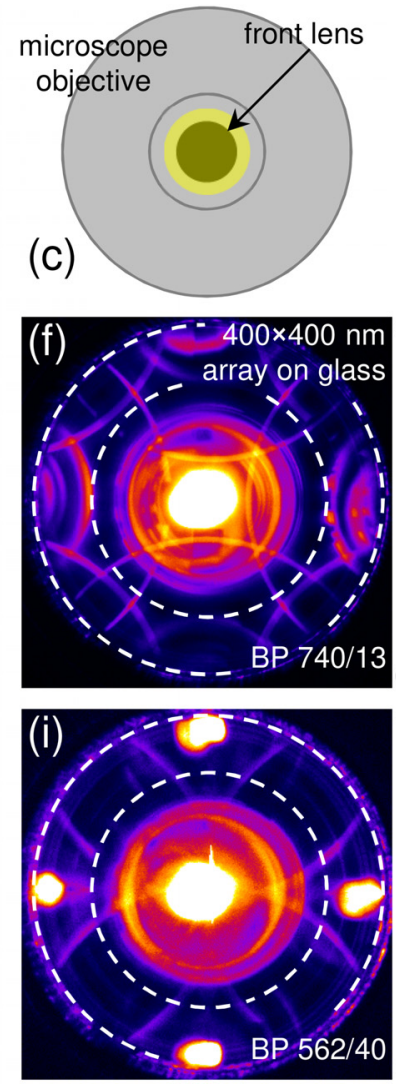

(d)
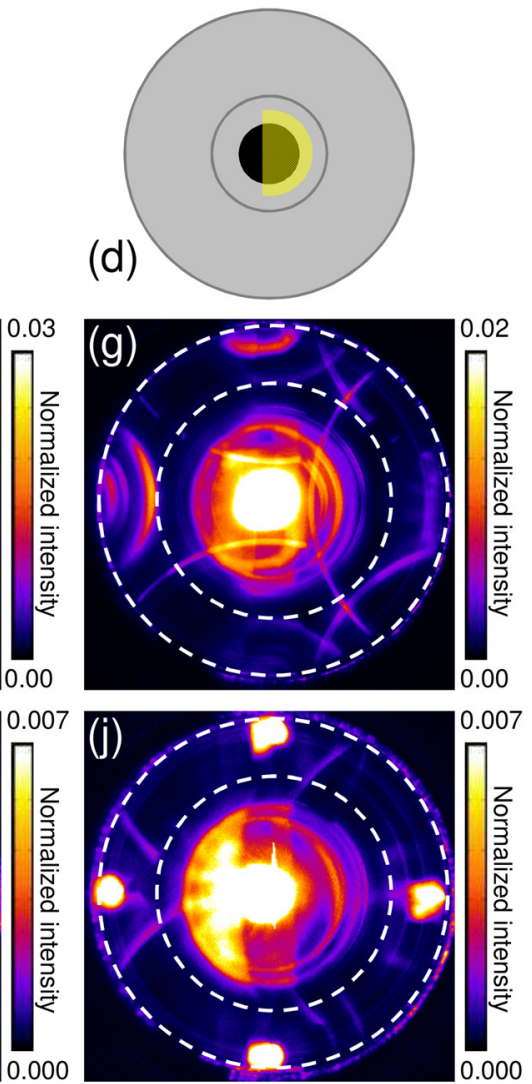

(e)
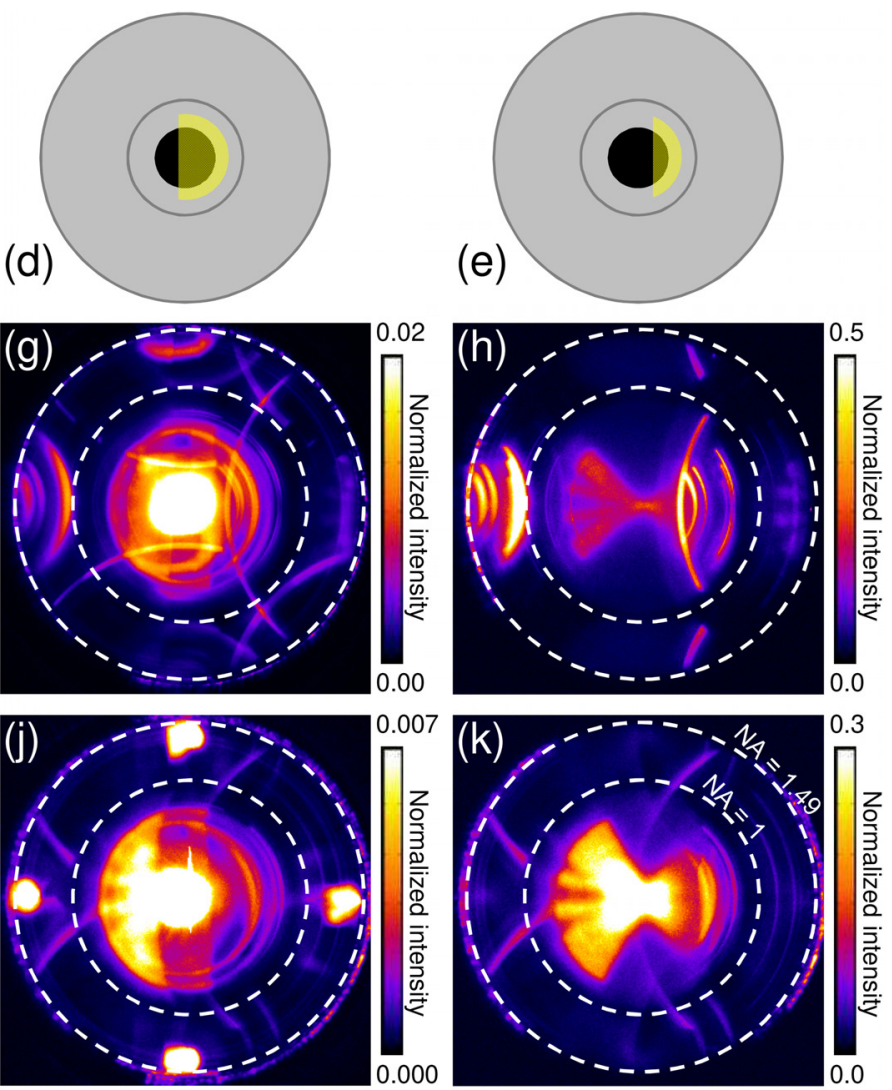

FIG. 5. The origin of the "condenser effect" revealed. (a) Schematics of the experiment: a transmitted light illumination configuration using a collimated white light beam with normal incidence is used, as shown in Fig. 2(c). The incident beam diameter exceeds the front lens diameter. A beam block is inserted between the primary light source and the sample (not shown) in order to truncate the incident light beam so that only part of the lens edge is illuminated. (b) Schematics of the Fourier-space image formation on the CCD camera: a pair of lenses projects an image of the objective's back focal plane onto the detector. Here, an incident light beam coming from the right yields a spot on the right side in the Fourier-space image. (c)-(e) Top-view schematics of the microscope objective, showing the front lens and the shape of the incident light beam: non-truncated in (c), less than half-truncated in (d), and more than half-truncated in (e). (f)-(k) Fourier-space optical microscopy images of a $400 \times 400 \mathrm{~nm}$ periodic, gold nanodot array on bare glass. Bandpass filters centered on wavelengths 740 nm (13 nm bandwidth) and $562 \mathrm{~nm}$ (40 nm bandwidth) are used in [(f)-(h)] and [(i)-(k)], respectively. The illumination corresponds to that shown in (c)-(e). In all images, the intensity scale is normalized with respect to the maximum of intensity in the image.

periodic array and collected by the lens. In this case, the involved wavevector combination is symmetrical to the one shown in Fig. 6(c) with respect to $\mathbf{k}_{\mathbf{y}}$. Therefore, the two possible optical pathways proposed in Fig. 6 result in light emitted at opposite angles $\theta$ and $-\theta$, respectively. Thus, by measuring the direction of the diffracted beam in Fourier space, the dominant mechanism may be determined.

Due to the axial symmetry of the problem with respect to the optical axis, the demonstration may only be carried out when the lens mount is not fully illuminated; part of the incident beam must be blocked. In Fig. 5(h), only a small area on the right-hand side of the front lens is illuminated. An incomplete circle is visible on the right side in Fourier space, which, according to Fig. 5(b), indicates that the scattered light from the array propagates from the right-hand side of the optical axis. In contrast, no such incomplete circle is visible on the left-hand side in Fourier space. In other words, scattered light from the array is only collected from the side of the optical axis that is illuminated, as in Fig. 6(b). Thus, the model introduced in Fig. 6(a) is ruled out, and the effect of multiple internal reflections inside the front lens is confirmed. The model of Fig. 6(b) also implies that the scattering of the incident light at the lens edge occurs essentially in the forward direction as is expected from the theory of light scattering from a circular aperture of diameter much larger than the wavelength.

We now explain why the "condenser effect" does not occur on metal-coated substrates. Figure 7 shows Fourierspace images of a $400 \times 400 \mathrm{~nm}$ periodic gold nanodot array on bare glass and on gold films deposited on glass. All images are obtained in the same transmitted light illumination configuration as used in Figs. 4 and 5. As seen in Fig. 7(a), the diffraction pattern in Fourier space of a nanostructure array on bare glass is very similar to the result for a nanostructure array on ITO-coated glass [Fig. 4(a)]. The two Fourier-space images have the same diffraction spots and a clover-shaped feature which is ascribed to the "condenser effect." On both substrates, the scattered light from the edges of the front lens illuminates the array from the substrate side, as described in Fig. 6(b), with an angle that is equal or slightly larger than the angular aperture of the objective. Liquid-immersion objectives generally have NA $>1$; therefore, the light from the lens edge undergoes total internal reflection in the substrate, and it is the resulting evanescent field at the surface of the substrate that interacts with the nanostructure array. 

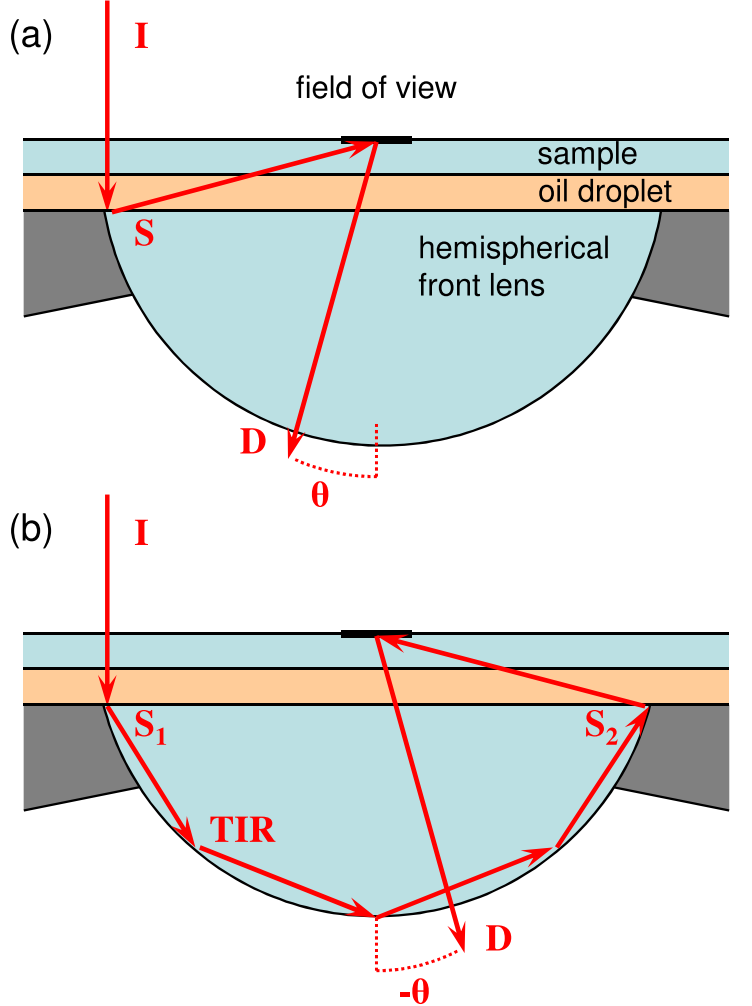

(c) $\circ$

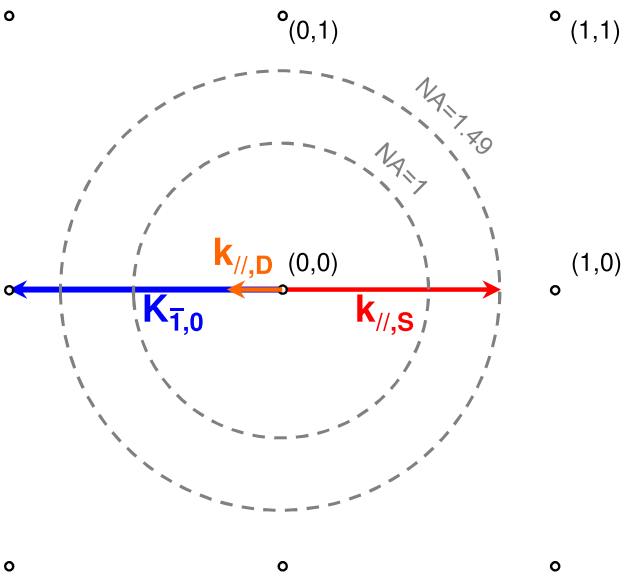

FIG. 6. (a) and (b) Side-view schematics of the experiment describing two possible scenarios for the "condenser effect". In (a), the incident light (I) scatters at the aperture of the metallic part holding the front lens of the objective. Part of the backscattered light (S) diffracts on the periodic array within the field of view and is collected (D) at an angle $\theta$. In (b), the scattered light $\left(\mathrm{S}_{1}\right)$ from the aperture undergoes a number of total internal reflections (TIR) inside the front lens before it scatters out $\left(S_{2}\right)$ in the upper medium. Part of this light diffracts on the periodic array and is collected (D) with an angle $-\theta$. (c) Fourier-space model for the scenario shown in (a), where $\mathbf{K}_{\overline{\mathbf{1}}, \mathbf{0}}$ is a Bragg vector of the periodic array and $\mathbf{k}_{\|, \mathbf{S}}$ and $\mathbf{k}_{\| . \mathbf{D}}=\mathbf{k}_{\|, \mathbf{S}}+\mathbf{K}_{\overline{\mathbf{1}, 0}}$ are the in-plane wavevector components of the scattered light from the aperture, before and after diffraction at the periodic array, respectively. The spots correspond to the expected diffraction pattern upon normal incidence. A $400 \times 400 \mathrm{~nm}$ periodic array and a vacuum wavelength $\lambda_{0}=740 \mathrm{~nm}$ are considered, as in Figs. $5(\mathrm{f})-5(\mathrm{~h})$.

As shown in Fig. 7, similar diffraction spots occur when the array is on a substrate that is coated with a $30 \mathrm{~nm}$ or $50 \mathrm{~nm}$-thick gold film, as compared to the same array on bare glass; however, the features of the "condenser effect" are absent on the metal-coated substrates. When the

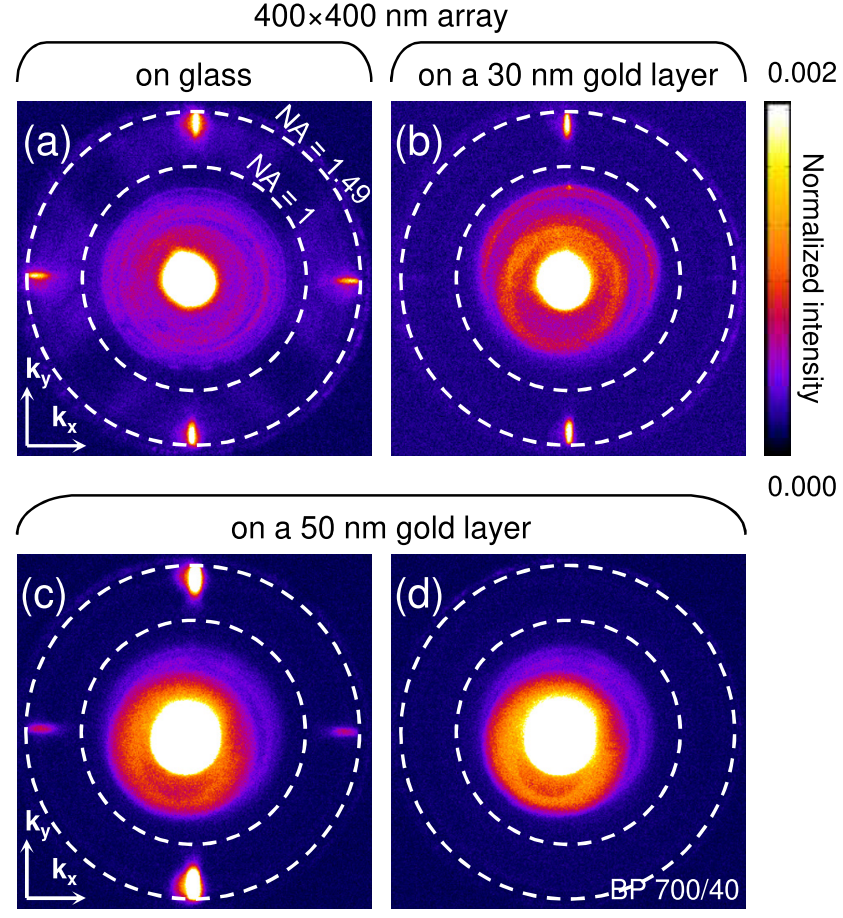

FIG. 7. Fourier-space optical microscopy images of a $400 \times 400 \mathrm{~nm}$ periodic gold nanodot array on bare glass or on gold films. All images are obtained in a transmitted light illumination configuration using a collimated white light beam with normal incidence, as shown in Fig. 2(c). A bandpass filter with a $40 \mathrm{~nm}$ bandwidth centered on a wavelength of $700 \mathrm{~nm}$ is used in (d). The intensity scale shown on the right, which is normalized with respect to the $(0,0)$ spot intensity, applies to all four images.

metallic film is thin enough, the incident light beam from the air side is partly transmitted and can follow the optical pathway described in Fig. 6(b); however, the transmission of the light scattered at a high angle from the lens edge $\left[\mathrm{S}_{2}\right.$ in Fig. 6(b)] is minimal in the case of a metallic film. Only if the scattered light couples coherently to surface plasmons at the metal-air interface will the transmission be significant enough to interact with the array. This only occurs for a very narrow angular range around the surface plasmon resonance (SPR) angle. At any other high angle, the evanescent field that is generated at the array upon internal reflection of light in the substrate is extremely weak, as compared to that at the SPR angle where the incident light and surface plasmon polaritons (SPPs) have matched in-plane wavevector components. ${ }^{62}$ Due to the SPP dispersion relation at the metal-air interface, the SPR angle is close to the critical angle of the substrate-air interface, which is lower than the angular aperture of most liquid-immersion objectives. Thus, the array on the sample does not "feel" the light scattered from the edge of the front lens and the "condenser effect" does not occur.

A noteworthy feature of the Fourier-space images in Figs. 7(b)-7(d) is the absence of the sharp circular ring that is expected for SPP leakage radiation in the substrate. Despite the presence of nanostructures on the metallic films, the incident light, which excites a significant portion of the array and not just a single nanodot, does not excite SPP waves at the metal-air interface. This is due to wavevector mismatching. SPPs at the metal-air interface have a larger 
wavevector than photons in air; therefore, a supplementary in-plane wavevector component is necessary for coherent coupling. In principle, the Bragg vectors $\mathbf{K}_{\mathbf{i}, \mathbf{j}}$ of the periodic array may be used to achieve wavevector matching; however, the Bragg vectors available from a $400 \times 400 \mathrm{~nm}$ periodic array are too large for any combination of them to match a circle of radius $\mathbf{k}_{\text {SPP }}$ within the spectral range where SPPs exist on an air-gold interface.

\section{Quantitative analysis of the artifacts}

Here, we use the Fourier-space images shown in Fig. 4 to carry out a quantitative analysis of the artifacts that occur under diascopic illumination, i.e., when the incident light hits the front aperture of the microscope objective. The intensity of the artifactual rings seen in Fig. 4 is two to three orders of magnitude lower than that of the $(0,0)$ spot and depends on wavelength (the longer the wavelength, the higher the relative intensity). The intensity of this artifact is one to two orders of magnitude lower than the $( \pm 1,0)$ and $(0, \pm 1)$ diffraction spots of the nanodot array. Nonetheless, once integrated over all collection angles, the relative contribution of all artifacts to the total amount of light transmitted in the microscope objective is seen to be significant. This may be calculated from Fig. 4 by integrating the Fourierspace images [after normalization with respect to the $(0,0)$ spot intensity] and subtracting the results obtained from Figs. 4(e) to 4(h), where no such artifacts occur, from those obtained from Figs. 4(a) to 4(d), respectively. In this way, we find that up to $35 \%$ of the total transmitted light from the nanodot array is due to artifacts; this result depends on the wavelength, with a contribution of $6 \%$ found at $650 \mathrm{~nm}$ and $35 \%$ at $740 \mathrm{~nm}$. The consequences that this result may have on spectroscopic measurements are discussed in Sec. III D.

The "condenser effect," i.e., the off-centered rings of diameter $k_{\|} / k_{0} \approx$ NA whose origin is explained in Fig. 6, is not the only contribution to the artificial increase in collected light described above. Centered on the $(0,0)$ spot, an additional ring and disk of radii $k_{\|} / k_{0}=0.65$ and 0.81 , respectively (i.e., $25^{\circ}$ and $32^{\circ}$ ), are observed, e.g., in Figs. 4(a)-4(d). These additional features cannot be ascribed to a reflection on the front side of the illumination source since the latter has an angular size viewed from the microscope objective that is about $10^{\circ}$. Instead, the scattered light from the objective's front aperture must be reflected back to the sample, as in Fig. 6, yet from other interfaces which are further inside the objective or at the tube lens of the microscope. Without the array [see Fig. 4(d)], only the centered ring and disk described above may be observed, and we find that they represent $19 \%$ of the total detected light. Therefore, we infer that, since the transmitted light from artifacts represents $35 \%$ of the total in Fig. 4(a), the "condenser effect" alone represents about $16 \%$ of the total transmitted light.

Additional results may be found in the supplementary material where similar experiments are performed on the same sample and under the same illumination conditions but using a different microscope objective, i.e., an Olympus PlanApoN $60 \times 1.45$ NA TIRF objective. These results show that the artifacts described above are not specific to a particular objective.
Similar off-centered rings are observed, yet with a smaller radius due to the lower NA, and their intensity is also about $0.1 \%$ of that of the $(0,0)$ spot. A centered disk of light is also observed.

\section{The "negative extinction" artifact}

We now describe a consequence of the "condenser effect." This effect can lead to significant artifacts in spectroscopic studies on extraordinary light transmission and collective optical effects in periodic arrays of nanostructures (nanoholes or nanoparticles) when using a microscope objective for collecting the transmitted light. As described in Sec. III B, the presence of a periodic array on the sample virtually extends the NA of the microscope objective as compared to the same substrate without any surface structuring. Thus, as a result of the array and the "condenser effect," there is an apparent increase in the transmission of light. We therefore refer to this effect as a "negative extinction" artifact.

Figure 8 shows the optical extinction spectra obtained from a $400 \times 400 \mathrm{~nm}$ periodic gold nanodot array on bare glass. All measurements are conducted in the same transmitted light illumination configuration as used in Figs. 4-7. Here, the extinction coefficient is defined relative to that of a bare air-glass interface, which has an extinction coefficient of $\approx 0.04$, essentially due to reflection. (In other words, the reported extinction coefficient $E$ is defined as $E=1-T$ with $T=\frac{I}{I_{0}}$ and $I$ the measured intensity with the sample and $I_{0}$ the measured intensity for a bare glass substrate). As in Fig. 4, we compare the results obtained when the width of the incident light beam is smaller and larger than the diameter of the front aperture of the objective. The two spectra in Fig. 8 exhibit the same spectral features which are due to the interplay of the localized surface plasmon resonances (LSPRs) in the nanoparticles and collective resonances in the array $^{13-15}$ and to the far-field interference of the transmitted and diffracted beams on the detector. Note that in the case

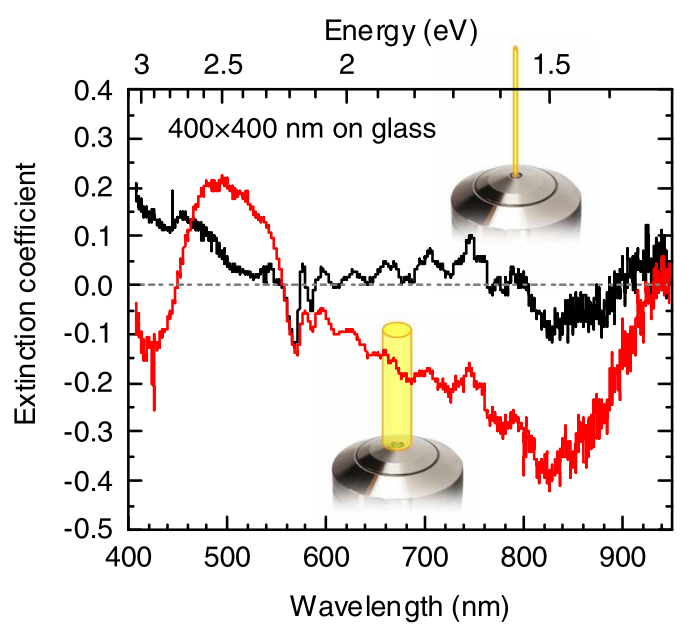

FIG. 8. Transmission spectra of a $400 \times 400 \mathrm{~nm}$ periodic gold nanodot array on bare glass. Both spectra are obtained in a transmitted light illumination configuration using a collimated white light beam with normal incidence, as shown in Fig. 2(c). The real-space image is projected on the entrance slit of the spectrometer. The width of the incident light beam is either smaller (black curve) or larger (red curve) than the diameter of the front aperture of the microscope objective. 
where the incident beam interacts with the edge of the front lens, the extinction coefficient decreases artificially with the wavelength for vacuum wavelengths of around $580 \mathrm{~nm}$ to $820 \mathrm{~nm}$. This reveals that a significant amount of light (up to a $40 \%$ increase in transmitted intensity at $\lambda_{0} \approx 820 \mathrm{~nm}$ ) enters the objective through the pathway described in Fig. 6(b). This relative amount is frequency dependent. Preventing the illumination of the edges of the front lens is thus mandatory for avoiding this "negative extinction" artifact and for conducting reliable spectroscopic measurements using an optical microscope.

In addition, the effects due to the interference of the transmitted and diffracted beams in the far-field may also be avoided by combining the $k$-space optical microscope with an imaging spectrometer. Projecting the Fourier-space image on the entrance slit of the spectrometer provides combined angular and spectral resolution; ${ }^{58}$ thus, the spectral content of the $(0,0)$ and $(i, j) \neq(0,0)$ diffraction orders may be separately analyzed. Further information is obtained in this way, as compared to traditional on-axis spectroscopic measurements on an optic bench or using a commercial spectrophotometer (i.e., no high angle objective lens is used), where only the $(0,0)$ order is generally detected.

\section{E. SPP-nanostructure array coupling}

In the following, we report on the advantages of $k$-space optical microscopy when applied to nanostructure arrays on substrates that support surface waves, in particular SPP waves on thin metallic films. Such plasmonic systems are of increasing interest in the growing field of sensing for their combined properties inherited from the local surface plasmon resonances in the nanostructures and the propagating surface waves on the underlying interface. ${ }^{46,47,63,64}$ This geometry notably allows one to combine surface enhanced emission processes and surface plasmon resonance sensing, two methods that have already been separately implemented in existing biochemical sensors. ${ }^{53,54}$ Below, we show how $k$ space optical microscopy may be used to investigate the coupling between a nanostructure array and the underlying metallic film. In particular, the angular dependence of this coupling and the resulting modification of the SPP dispersion relation are investigated. As shown below, our results confirm well-known grating diffraction laws and existing results in optical spectroscopy and angle-resolved microscopy of periodic systems, as described in, e.g., Refs. 36-38 and 41-43 and reviewed in Ref. 9.

\section{Forbidden regions or gaps}

Figure 9 shows the Fourier-space images obtained from periodic gold nanodot arrays on a 50-nm thick gold layer on glass illuminated in the reflected light configuration with a focused laser beam as presented in Fig. 2(a). Unlike in Fig. 3 , here we use a spinning diffuser to blur the speckle of the laser beam in order to improve the visibility of the current features of interest in Fourier space. The orientation of the linear polarization of the incident light is specified in the top right corner of the images. Data measured on a $400 \times 400 \mathrm{~nm}$ and a $500 \times 500 \mathrm{~nm}$ periodic array are shown in Figs. 9(a),
$400 \times 400 \mathrm{~nm}$ array on a $50 \mathrm{~nm}$ gold film
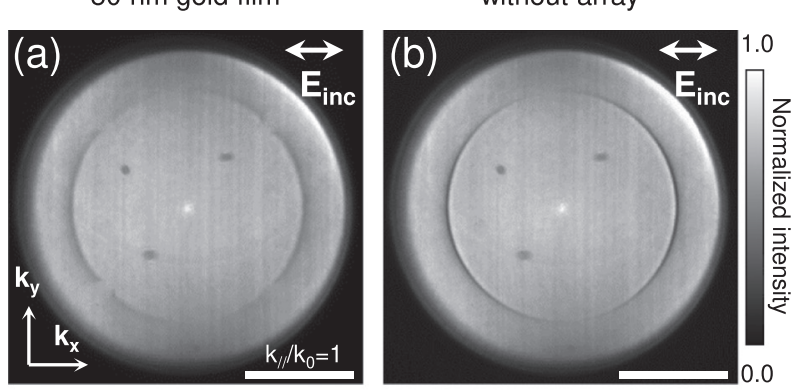

$500 \times 500 \mathrm{~nm}$ array on a $50 \mathrm{~nm}$ gold film
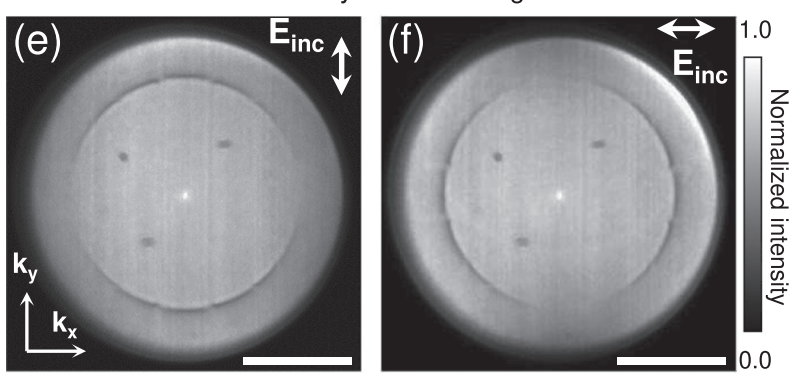

$50 \mathrm{~nm}$ gold film without array
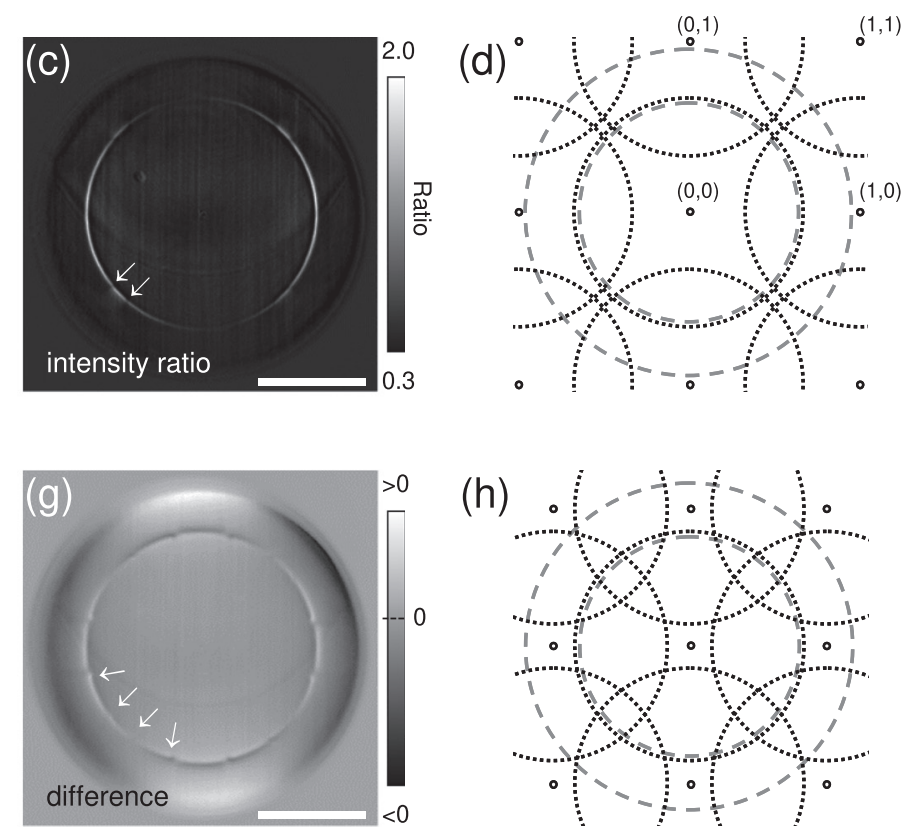

(h)

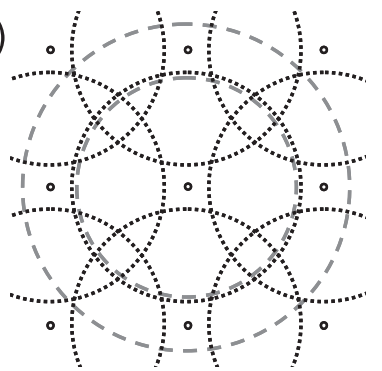

FIG. 9. Fourier-space optical microscopy images of periodic gold nanodot arrays on a 50-nm thick gold layer. All images are obtained in a reflected light illumination configuration using a focused laser beam, as shown in Fig. 2(a). White double arrows in the right-hand corners of the images indicate the polarization of the incident light. [(a)-(d)] Intensity images measured on (a) a $400 \times 400 \mathrm{~nm}$ array and (b) a structureless area of the gold film and (c) their intensity ratio. [(e)-(h)] Intensity images measured on a $500 \times 500 \mathrm{~nm}$ array with the incident field parallel to (e) the $\mathbf{k}_{\mathbf{y}}$ and (f) $\mathbf{k}_{\mathbf{x}}$ axes and (g) the subtraction of one image from the other. Parts (d) and (h) show sketches of circles with a radius corresponding to the SPP wavevector and centered on the diffraction orders. Forbidden regions or gaps are expected to occur in the SPP dispersion relation when these circles intersect the one centered on $(0,0)$. The two dashed circles have a radius of $k_{\|} / k_{0}=1.0$ or 1.49 , respectively. Part (d) corresponds to the $400 \times 400 \mathrm{~nm}$ array and part (h) to the $500 \times 500 \mathrm{~nm}$ array. In the images shown in (a), (b), (e), and (f), the intensity scale is normalized with respect to the intensity maximum. The scale bar indicates the Fourier-space calibration $k_{\|} / k_{0}=1$. 
9(e), and 9(f). Two different methods of data presentation are used. In the first case, a Fourier-space image is first recorded by illuminating the array, a second image is obtained with the illumination of a structureless area, and the intensity ratio, shown in Fig. 9(c) is obtained by dividing the first image by the second. Due to additional SPP scattering losses, the SPR is weaker and angularly broader on the array than on the structureless area of the metallic film. This yields a sharp ring in the intensity ratio image. In the second case, the Fourier-space images recorded on the array with the two orthogonal incident light polarizations are subtracted one from the other, yielding Fig. 9(g).

Both image processing methods used in Fig. 9 reveal discontinuities in the ring of radius $k_{\|}=k_{S P P}$ (see the white arrows in the optical images). These discontinuities correspond to the intersection of the isofrequency curves, i.e., the replica of the SPP ring centered on the diffraction spots of the $400 \times 400 \mathrm{~nm}$ and $500 \times 500 \mathrm{~nm}$ periodic arrays, as shown in the Fourier-space models in Figs. 9(d) and 9(h), respectively. In particular, gaps in the SPP dispersion relation, ${ }^{36,65}$ i.e., angular domains of forbidden SPP propagation, around specific directions of the $\mathbf{x y}$ plane are visible. Gap openings on periodically structured metallic surfaces have been used to control the spontaneous emission of quantum emitters in the near field of the surface, ${ }^{37,38}$ and SPP beam self-collimation, steering, and negative refraction ${ }^{42,43}$ have been obtained. Here, $k$-space optical microscopy leads to the characterization of several phenomena in a single image capture, e.g., the interaction between the incident light, the metallic film, and the periodic array and the effect of the film-array coupling on the SPP dispersion relation. These measurements are obtained simultaneously at all angles of incidence within the objective aperture and for both $p$ and $s$ polarizations.

\section{Angular resonances}

Figure 10 shows Fourier-space images of a $500 \times 500 \mathrm{~nm}$ periodic gold nanodot array on a 50-nm thick gold layer measured upon reflection of a collimated laser beam for two different angles of incidence $\theta_{\text {inc }}$ (additional experimental details can be found in the supplementary material). This illumination configuration is used to investigate the angular dependence of the coupling of the incident light with the nanostructure array. The results obtained from the array [Figs. 10(b) and 10(d)] are compared to the results obtained from featureless areas of the gold layer, i.e., far from the nanostructures [Figs. 10(a) and 10(c)].

At normal incidence $\theta_{i n c}=0\left(k_{\|}=0\right)$, the Fourier-space image exhibits a single spot corresponding to the reflection of the incident light [i.e., the $(0,0)$ spot of the diffraction pattern], irrespective of whether the surface of the metallic film is periodically structured or not. The faint spot at the position of the asterisk in Figs. 10(a)-10(d) is an artifact due to a back reflection inside the microscope and is not related to the geometry of the sample. Apart from this artifact, the $(0,0)$ spot is the only visible feature in Fourier space within the accessible angular range.

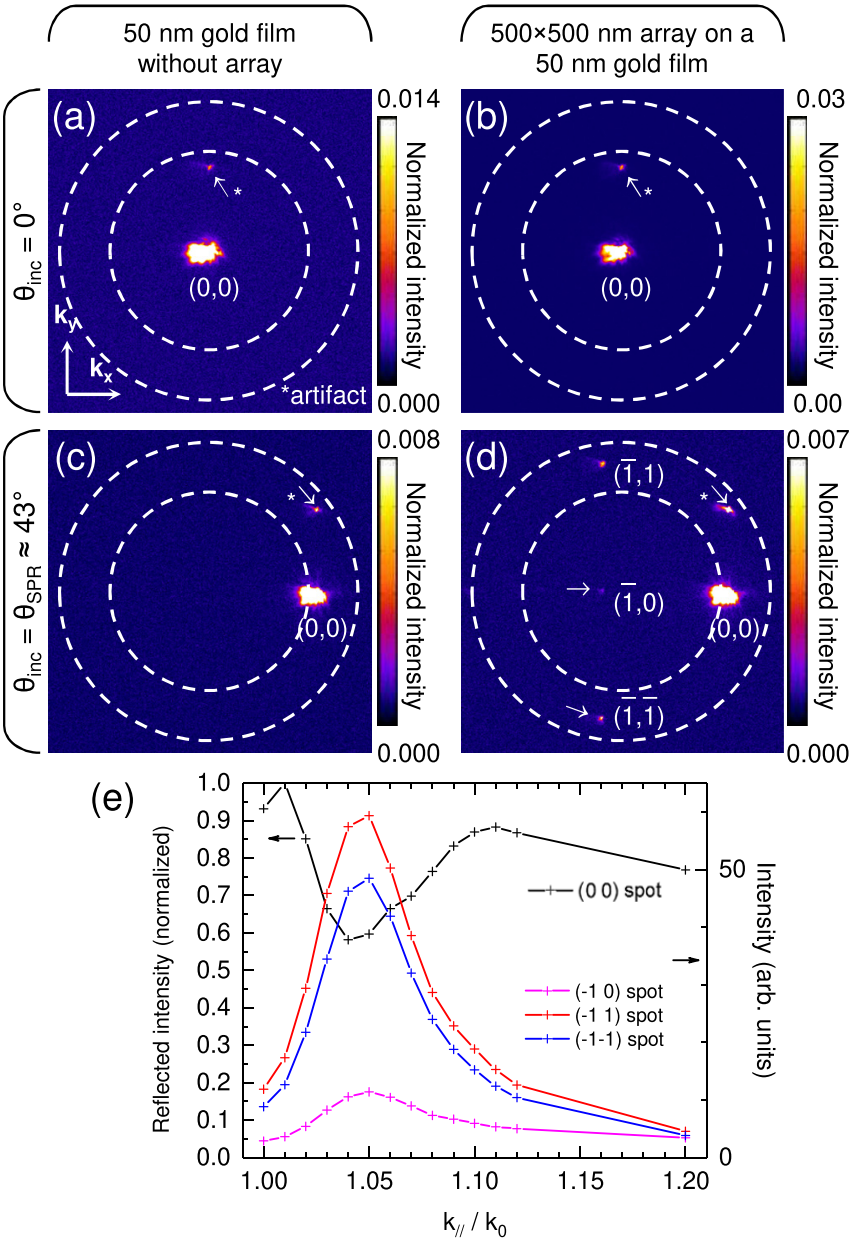

FIG. 10. Fourier-space optical microscopy images of a $500 \times 500 \mathrm{~nm}$ periodic gold nanodot array on a 50-nm thick gold layer. All images are obtained in a reflected light illumination configuration using a collimated laser beam with a variable angle of incidence (see details in the supplementary material). Illumination is either [(a) and (b)] in normal incidence $\left(\theta_{\text {inc }}=0^{\circ}\right)$ or $[(\mathrm{c})$ and (d)] at an angle of incidence, allowing light-SPP wavevector matching $\left(\theta_{\text {inc }}=\theta_{S P R} \approx 43^{\circ}\right)$. Images in [(a) and (c)] and [(b) and (d)] are measured on a structureless area and a $500 \times 500 \mathrm{~nm}$ array on the gold film, respectively. In all images, the intensity scale is normalized with respect to the $(0,0)$ spot intensity. (e) Intensity of the light reflected and scattered from a $500 \times 500 \mathrm{~nm}$ array on the gold film, as a function of the in-plane wavevector of the incident light, obtained from the $(0,0)$ and $( \pm 1, \pm 1)$ diffraction spots, respectively. The relationship between the incident angle and the in-plane wavevector component is $k_{\|} / k_{0}=n_{\text {glass }} \sin \theta_{\text {inc }}$. The data shown in (e) are corrected for the $(\cos \theta)^{-1}$ apodization factor, i.e., the experimental intensity values are multiplied by $\cos \theta$. The reflected intensity (black curve), obtained from the $(0,0)$ spot, is normalized with respect to its maximum value within the investigated angular range.

It is only when $\theta_{\text {inc }}$ is close to $\theta_{S P R} \approx 43^{\circ}\left(k_{\|}=k_{S P P}\right)$ that other spots from the diffraction pattern of the periodic array are visible in the image [see Fig. 10(d)]. This occurs when there is matching between the in-plane wavevectors of the incident light and the SPP waves at the air-metal interface. Resonant light-to-SPP coupling strongly enhances the transmitted (evanescent) field which is otherwise comparatively very weak when a 50 -nm thick gold layer on glass is illuminated off-resonance. ${ }^{62}$ Since it is the transmitted field that diffracts at the periodic array, the intensity of the diffraction spots $(i, j) \neq(0,0)$ is related to the excited SPP intensity. Thus, two different types of analysis are possible. The intensity variation of the diffraction spots versus $\theta_{i n c}$ may be 
used to study the angular dependence of the internal reflection coefficient by monitoring the intensity of the $(0,0)$ spot. Also, the angular dependence of the SPP intensity at the airmetal interface may be determined by monitoring the other diffraction spots. The result of such an analysis is shown in Fig. 10(e). The spot intensities are plotted as a function of $k_{\|} / k_{0}=n_{\text {glass }} \sin \theta_{\text {inc }}$ for the angular range of $41^{\circ}\left(k_{\|} / k_{0}\right.$ $=1)$ to $52^{\circ}\left(k_{\|} / k_{0}=1.2\right)$. The reflection coefficient and the diffraction spot intensities exhibit a dip and a peak, respectively, for the displayed $1.04<k_{\|} / k_{0}<1.05$ range $\left(43.2^{\circ}<\theta_{\text {inc }}<43.8^{\circ}\right)$, with the same full width at half maximum of $0.054\left(2.8^{\circ}\right)$. The attenuated total reflection (ATR) angle coincides almost perfectly with the SPR angle as expected for a Kretschmann-Raether experiment. ${ }^{66}$ In this way, $k$-space optical microscopy provides an alternative technique to prism-based experiments on an optic bench for SPR measurements.

In addition, by controlling the incident light polarization, $k$-space optical microscopy may be used for quantitative angle-resolved reflectivity measurements, i.e., a sort of micro-ellipsometry. This is similar to an already known and implemented technique known as Mueller polarimetry ${ }^{67}$ or Mueller matrix imaging ellipsometry. ${ }^{68}$ Importantly, reflected light images may only be converted into quantitative reflectance maps if they can be normalized by the incident light intensity. The incident light intensity may be obtained from measurements performed under the same illumination conditions on a totally reflective interface. For instance, a gold film thicker than typically $200 \mathrm{~nm}$ on glass could be used to obtain the necessary reference images.

\section{F. Accurate lateral and axial positioning of the sample}

In this section, we report on the possibility of applying $k$ space optical microscopy to the problem of accurate lateral and axial positioning of a sample or a laser spot in an optical microscope. In particular, the application proposed below concerns (but is not limited to) the control of the absolute position of the sample in an optical microscope or the relative position of a laser spot with respect to the sample. The data discussed in Fig. 11 are obtained under the same illumination conditions as used in Fig. 3(g), i.e., reflected light illumination using a focused laser beam as described in Fig. 2(a).

Figure 11 shows a series of three Fourier-space images measured on a $400 \times 400 \mathrm{~nm}$ periodic array of gold nanodots on bare glass. The relative lateral position of the laser spot with respect to the array on the sample is different for each image. In order to acquire this series, the position of the laser spot is laterally shifted in steps of $100 \mathrm{~nm}$ along the $\mathbf{x}$-axis in real space (the sample is translated using the piezoelectric stage, and the laser spot is kept fixed). We observe that such a lateral shift, that is but a fraction of the array period, results in a significant modification of the intensity distribution in Fourier space. Namely, it is the intensity ratio between the left $\left(k_{x}<0\right)$ and right $\left(k_{x}>0\right)$ halves of the Fourier-space image that changes. A lateral shift in real space corresponds to the addition of a constant phase ramp, i.e., a tilt of the wave front, in Fourier space. This phase modification yields an intensity change since we record the interference pattern
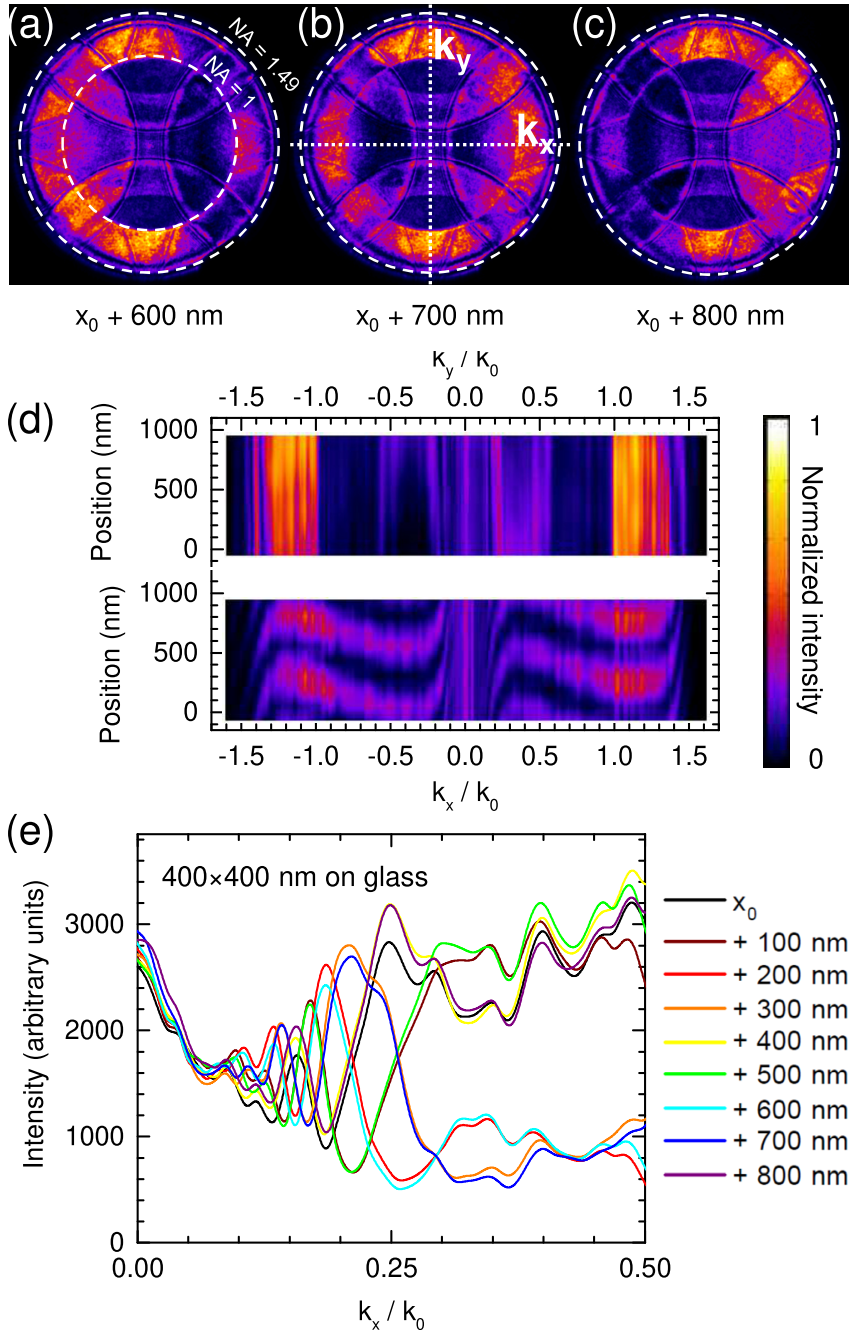

FIG. 11. Fourier-space optical microscopy images of a $400 \times 400 \mathrm{~nm}$ periodic gold nanodot array on bare glass. All images are obtained in a reflected light illumination configuration using a focused laser beam, as shown in Fig. 2(a). A nanopositioning piezo stage is used to control the lateral position of the sample and thus the relative position of the laser spot with respect to the nanodot array. [(a)-(c)] Images obtained for three different lateral positions of the laser spot with respect to the array. The position is changed by $100 \mathrm{~nm}$ along the $x$ axis for each subsequent image. (d) 2D maps of the intensity variation along the $\mathbf{k}_{\mathbf{x}}$ and $\mathbf{k}_{\mathbf{y}}$ axes in Fourier space as a function of the lateral shift of the sample position (in steps of $100 \mathrm{~nm}$ ) retrieved from the intensity profiles of parts [(a)-(c)]. Profiles from along the $\mathbf{k}_{\mathbf{x}}$-axis in part (d) are plotted in (e).

of the specular reflection and the first-order diffraction of the incident beam. The optical path difference between the reflected beam $(0,0)$ and the four first-order diffracted beams $(\overline{1}, 0),(0, \overline{1}),(1,0)$, and $(0,1)$ is only the same when the laser spot is centered on one of the nanostructures in the array as in Fig. 11(b). In this case, only the $\mathbf{k}_{\mathbf{x}} / \mathbf{k}_{\mathbf{y}}$ anisotropy due to the linear polarization of the incident light may be seen in Fourier space. As soon as the symmetry of the beamarray system is broken, the intensity distribution in Fourier space becomes clearly asymmetric with respect to the $\mathbf{k}_{\mathbf{y}}$ axis.

Intensity profiles taken from Fourier-space images acquired for different lateral shifts along the $\mathbf{x}$-axis are displayed in Fig. 11(d) using the same color scale as in the source images. As the sample is moved along the $\mathbf{x}$-axis, 
little change is seen along the $\mathbf{k}_{\mathbf{y}}$ direction in Fourier space [see the top half of Fig. 11(d)]. Looking along the $\mathbf{k}_{\mathbf{x}}$ direction, however [bottom half of Fig. 11(d)], the variation in intensity is clearly periodic with a lateral shift, and the period is equal to that of the nanostructure array. A restricted $k_{x^{-}}$ range of these profiles is plotted in Fig. 11(e). Here, we see that the intensity ratio between two points in Fourier space, e.g., $\left(k_{x}, k_{y}\right)=\left(0.20 k_{0}, 0\right)$ and $\left(0.25 k_{0}, 0\right)$, may be used to estimate or monitor the relative position of the laser spot with respect to the periodic array to a precision that is far beyond the Rayleigh criterion. This precision can descend to the sub- $100 \mathrm{~nm}$ or sub- $10 \mathrm{~nm}$ range, depending on the noise level of the experiment. In contrast, such accurate control in real-space typically requires a correlation analysis of the real-space images in order to determine the lateral shifts of the array and/or a super-localization algorithm that pinpoints and tracks the laser spot. Thus, $k$-space optical microscopy provides an appealing alternative to existing real-space techniques for the accurate lateral positioning of a laser spot or sample in an optical microscope. Similar techniques, referred to as interference Fourier scatterometry, were previously reported and applied to the nanoscopic lateral positioning and the characterization of sub-lambda features of 1D grating structures. ${ }^{69,70}$

The technique of $k$-space optical microscopy is also sensitive to shifts in the axial position of the sample, i.e., to the defocusing of a periodic array. This has potential applications for auto-focus systems in optical microscopy. Figure 12 shows a series of six real-space images of the same sample as in Fig. 11, obtained upon transmitted light illumination using a collimated white light beam in normal incidence [see schematics in Fig. 2(c)]. The relative axial position of the sample with respect to the objective lens is different for each image. The microscope objective is translated along the $\mathbf{z}$ axis, and the stage holding the sample is kept fixed; thus, the periodic array moves away from the front focal plane of the objective (referred to as $z_{0}$ ) and a defocused image is recorded. Figure 12(g) shows the $\mathbf{x z}$ and $\mathbf{y z}$ cuts in the stack of defocused images.

Figure $12(\mathrm{~g})$ reveals a periodic replication of the image of the array in the $\mathbf{z}$ direction, with periodic contrast inversion, especially when the array is axially located beyond the front focal plane of the objective $\left(z>z_{0}\right)$. This is due to the well-known Talbot effect ${ }^{71-73}$ (the fading of the replica beyond $5 \mu \mathrm{m}$ away from the focus is due to the broad spectrum of the illumination source). The Talbot effect increases the difficulty of finding the precise in-focus position of the sample along the axial direction when using only real-space images; this is even more so the case when sharper spatial features occur in the replica, as compared to the in-focus, real-space image of the array, as seen in Figs. 12(a) and 12(b). We demonstrate below that $k$-space optical microscopy constitutes an advantageous alternative to real-space image analysis for the accurate axial positioning of a periodically structured sample. Unambiguous determination of the image focus is possible in the case of Fourier-space imagery.

Figure 13 shows three Fourier-space images acquired for the same three sample positions along $\mathbf{z}$ as the real-space images shown in Figs. 12(d) to 12(f), yet measured in

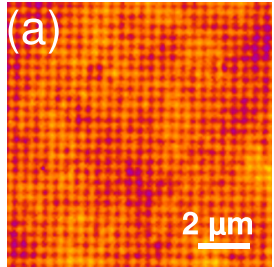

$\mathrm{z}_{0}$ (in focus)

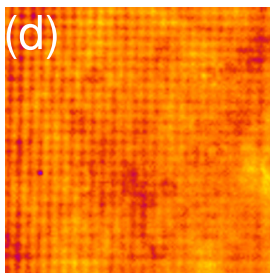

$\mathrm{z}_{0}-0.5 \mu \mathrm{m}$

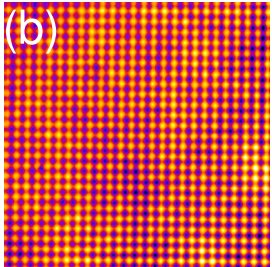

$z_{0}+1.5 \mu m$

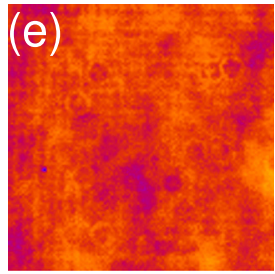

$\mathrm{z}_{0}-1.5 \mu \mathrm{m}$

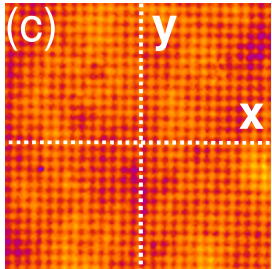

$z_{0}+3.5 \mu m$

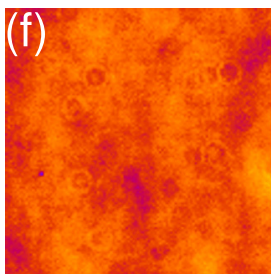

$\mathrm{z}_{0}-3.5 \mu \mathrm{m}$ (g)

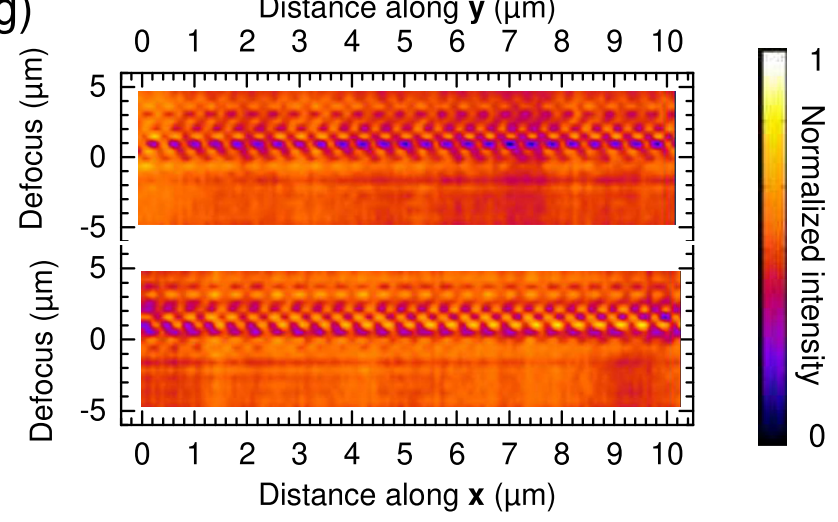

FIG. 12. Real-space optical microscopy images of a $400 \times 400 \mathrm{~nm}$ periodic gold nanodot array on bare glass. All images are obtained in a transmitted light illumination configuration using a collimated white light beam with normal incidence, as shown in Fig. 2(c). The fine focusing knob of the microscope stand is used to control the axial position of the objective and thus the relative axial position of the front focal plane with respect to the nanodot array. [(a)-(f)] Images obtained at six different axial positions of the objective. (g) 2D maps of the intensity variation along the $\mathbf{x}$ and $\mathbf{y}$ axes in real space upon axially shifting the objective position in steps of $0.5 \mu \mathrm{m}$. These data are retrieved from images such as those in part [(a)-(f)] along the $\mathbf{x}$ and $\mathbf{y}$ axes as shown in part (c).

reflection upon illumination with a focused laser beam, as in Fig. 11 [see also Fig. 2(a)]. In this illumination configuration, the laser beam is invariably focused on the front focal plane of the microscope objective. As the objective is axially translated, both the focal plane and the laser spot are moved away from the sample. In an optical microscope, the imaging conditions in Fourier space are compatible with the paraxial approximation (i.e., the emitted optical rays make low angles with respect to the optical axis). Therefore, it is commonly admitted that the Fourier-space image is comparatively less sensitive to sample defocusing than the real-space image. Nevertheless, the Fourier-space image can be highly sensitive to the defocusing of the illumination spot on the sample when the angular aperture of the focused beam is large. Moving the laser spot axially away from the periodic array increases the dephasing between the $k$-vector components of the incident field at the array (which are all in phase at the focus of the beam). As discussed in Sec. III A, $k$-space 


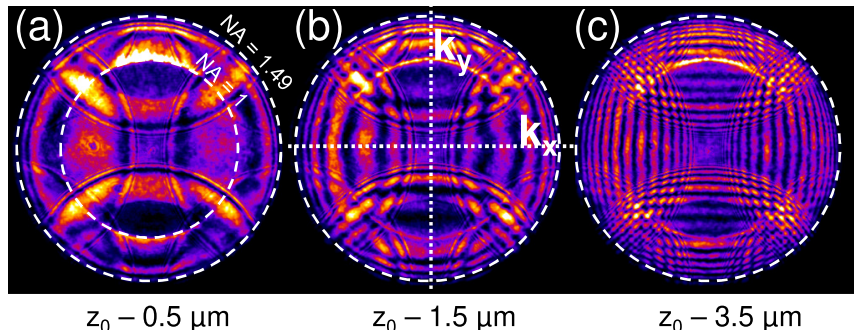

$\mathrm{z}_{0}-0.5 \mu \mathrm{m}$ $\mathrm{z}_{0}-1.5 \mu \mathrm{m}$ $\mathrm{z}_{0}-3.5 \mu \mathrm{m}$

(d)

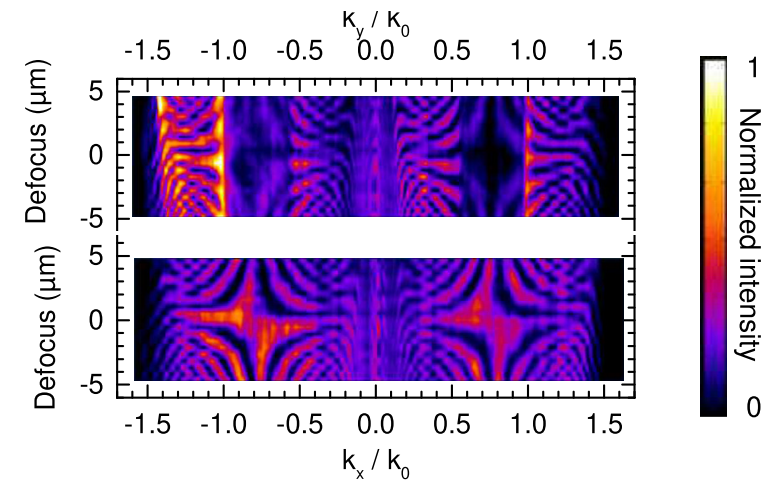

FIG. 13. Fourier-space optical microscopy images of a $400 \times 400 \mathrm{~nm}$ periodic gold nanodot array on bare glass. All images are obtained in a reflected light illumination configuration using a focused laser beam, as shown in Fig. 2(a). The fine focusing knob of the microscope stand is used to control the axial position of the objective and thus the relative axial position of the front focal plane and the laser spot (which remains focused on the front focal plane) with respect to the nanodot array. [(a)-(c)] Images obtained for three different axial positions of the objective. (d) 2D maps of the intensity variation along the $\mathbf{k}_{\mathbf{x}}$ and $\mathbf{k}_{\mathbf{y}}$ axes in Fourier space as a function of the axial shift of the sample (step size $0.5 \mu \mathrm{m}$ ). These data are retrieved from images such as those shown in parts [(a)-(c)] along the $\mathbf{k}_{\mathbf{x}}$ and $\mathbf{k}_{\mathbf{y}}$ axes as denoted in (b).

optical microscopy of periodic nanostructure arrays converts phase information from the back reflected light into intensity contrast in the Fourier-space image, through the interference of the specular reflection and first-order diffraction of the incident light beam. As shown in Fig. 13, a pattern of dark and bright fringes, with the same in-plane symmetry as the periodic array (i.e., quadratic in the present case), is seen in the $\mathbf{k}_{\mathbf{x}} \mathbf{k}_{\mathbf{y}}$ plane, while a hyperbolic fringe pattern is seen for the $\mathbf{k}_{\mathbf{x}}$ or $\mathbf{k}_{\mathbf{y}}$ cross-sections as a function of defocus. The number of visible fringes rapidly increases with the defocusing distance, in such a way that their number and position can be used to evaluate the defocusing distance to a precision that is far beyond the depth of focus.

The potential of $k$-space optical microscopy for autofocusing applications in optical microscopy still needs to be investigated further. ${ }^{74}$ Yet the technique presented here is, in principle, compatible with existing imaging techniques such as those based on sample photoluminescence or nonlinear coherent emission processes. In these cases, the excitation light propagating back from the filter cube of the microscope may be imaged in Fourier space on a remote camera, just by inserting a semi-reflective component in the light beam before the entrance port of the microscope. In this way, autofocusing using $k$-space optical microscopy may be implemented as a simple add-on module and only requires that part of the sample area in the field of view contains a periodic nanostructure array. Furthermore, a single module can be used for both accurate lateral and axial positioning since the same illumination configuration is used, and the two measurements are compatible; the lateral phase shift induced in Fourier space by a lateral position shift in real space is not expected to depend on the axial position of the array with respect to the focal plane and the focus spot.

\section{CONCLUSION}

Implementing $k$-space optical microscopy with the various illumination configurations available on an inverted optical microscope equipped with a liquid-immersion objective provides a powerful toolbox for the characterization of periodic samples, including nanostructure arrays with subwavelength periods. A number of effects occur in the formation of the Fourier-space image in an optical microscope, some of which are well known from the general theory of optical imaging systems while others are more unexpected and specific to the use of liquid-immersion objectives. As shown in the present study, understanding these effects leads to the extraction of more information about the sample from Fourier-space images and also to the identification of the origin of possible artificial results, e.g., in spectroscopic measurements.

Among the most interesting results are the $k$-space optical microscopy images of periodic samples illuminated with a focused, coherent light beam, in an episcopic configuration. These images result from the coherent interference of the reflected and diffracted light beams. Crucial phase information, which can be either related to the sample geometry or to the relative position of the illumination focus and the sample, is thus converted into intensity variations; this leads to possible applications for the precise control of the sample position in optical microscopy.

In addition, the illumination of periodic samples with a collimated light beam in a diascopic configuration offers novel possibilities in $k$-space optical microscopy. This is thanks to the "condenser effect." This artifact makes higher spatial frequencies accessible and reveals the geometry (i.e., the periods, orientation, and symmetry) of periodic samples beyond the resolution limit of the microscope objective (which is fixed by its NA). Moreover, the "condenser effect" may be controlled (or removed) by changing the incident beam diameter. This is because this artifact is the result of light scattering at the objective's front aperture. Thus, the same high-NA objective may be used both for imaging periodic samples with extended resolution and performing spectroscopic measurements without detrimental artifacts due to additional light entering the objective.

\section{SUPPLEMENTARY MATERIAL}

See supplementary material for additional experimental details, an additional discussion of the interpretation of Fourier-space images, and further investigations on the effect of the objective properties on the "condenser effect."

\section{ACKNOWLEDGMENTS}

We acknowledge the technical support from the micronanotechnology platform of the Center for Nanosciences and 
Nanotechnologies (C2N-CTU) in Orsay, France, and the financial support from the Région Ile-de-France in the framework of DIM Nano-K. We thank Dr. S. Lévêque-Fort for enlightening discussions and the loan of the Olympus objective.

${ }^{1}$ M. A. Lieb, J. M. Zavislan, and L. Novotny, "Single-molecule orientations determined by direct emission pattern imaging," J. Opt. Soc. Am. B 21, 1210-1215 (2004).

${ }^{2}$ J. A. Kurvits, M. Jiang, and R. Zia, "Comparative analysis of imaging configurations and objectives for Fourier microscopy," J. Opt. Soc. Am. A 32, 2082-2092 (2015).

${ }^{3}$ R. Biehl and T. Palberg, "Real space and Fourier microscopy of colloidal suspensions confined to a parallel plate geometry," Rev. Sci. Instrum. 75, 906-914 (2004).

${ }^{4}$ I. Sersic, C. Tuambilangana, and A. F. Koenderink, "Fourier microscopy of single plasmonic scatterers," New J. Phys. 13, 083019 (2011).

${ }^{5}$ J. F. Galisteo-López, M. López-García, A. Blanco, and C. López, "Studying light propagation in self-assembled hybrid photonic-plasmonic crystals by Fourier microscopy," Langmuir 28, 9174-9179 (2012).

${ }^{6}$ Y. Fontana, G. Grzela, E. P. A. M. Bakkers, and J. G. Rivas, "Mapping the directional emission of quasi-two-dimensional photonic crystals of semiconductor nanowires using Fourier microscopy,” Phys. Rev. B 86, 245303 (2012).

${ }^{7}$ G. Lozano, T. Barten, G. Grzela, and J. G. Rivas, "Directional absorption by phased arrays of plasmonic nanoantennae probed with time-reversed Fourier microscopy,” New J. Phys. 16, 013040 (2014).

${ }^{8} \mathrm{P}$. Arora and A. Krishnan, "Fourier plane colorimetric sensing using broadband imaging of surface plasmons and application to biosensing," J. Appl. Phys. 118, 233105 (2015).

${ }^{9}$ F. J. García de Abajo, "Colloquium: Light scattering by particle and hole arrays," Rev. Mod. Phys. 79, 1267-1290 (2007).

${ }^{10}$ B. Lamprecht, G. Schider, R. T. Lechner, H. Ditlbacher, J. R. Krenn, A. Leitner, and F. R. Aussenegg, "Metal nanoparticle gratings: Influence of dipolar particle interaction on the plasmon resonance," Phys. Rev. Lett. 84, 4721 (2000).

${ }^{11}$ S. Linden, J. Kuhl, and H. Giessen, "Controlling the interaction between light and gold nanoparticles: Selective suppression of extinction," Phys. Rev. Lett. 86, 4688-4691 (2001).

${ }^{12}$ V. G. Kravets, F. Schedin, and A. N. Grigorenko, "Extremely narrow plasmon resonances based on diffraction coupling of localized plasmons in arrays of metallic nanoparticles," Phys. Rev. Lett. 101, 087403 (2008).

${ }^{13} \mathrm{~B}$. Auguié and W. Barnes, "Collective resonances in gold nanoparticle arrays," Phys. Rev. Lett. 101, 143902 (2008).

${ }^{14} \mathrm{~B}$. Auguié and W. L. Barnes, "Diffractive coupling in gold nanoparticle arrays and the effect of disorder," Opt. Lett. 34, 401-403 (2009).

${ }^{15}$ B. Auguié, X. M. Bendaña, W. L. Barnes, and F. J. García de Abajo, "Diffractive arrays of gold nanoparticles near an interface: Critical role of the substrate," Phys. Rev. B 82, 155447 (2010).

${ }^{16}$ A. G. Nikitin, A. V. Kabashin, and H. Dallaporta, "Plasmonic resonances in diffractive arrays of gold nanoantennas: Near and far field effects," Opt. Express 20, 27941-27952 (2012).

${ }^{17}$ A. G. Nikitin, T. Nguyen, and H. Dallaporta, "Narrow plasmon resonances in diffractive arrays of gold nanoparticles in asymmetric environment: Experimental studies," Appl. Phys. Lett. 102, 221116 (2013).

${ }^{18}$ L. Langguth, D. Punj, J. Wenger, and A. F. Koenderink, "Plasmonic band structure controls single-molecule fluorescence," ACS Nano 7, 8840-8848 (2013).

${ }^{19}$ G. Lozano, G. Grzela, M. A. Verschuuren, M. Ramezani, and J. G. Rivas, "Tailor-made directional emission in nanoimprinted plasmonic-based light-emitting devices," Nanoscale 6, 9223-9229 (2014).

${ }^{20}$ L. Langguth, A. H. Schokker, K. Guo, and A. F. Koenderink, "Plasmonic phase-gradient metasurface for spontaneous emission control," Phys. Rev. B 92, 205401 (2015)

${ }^{21}$ D. K. G. de Boer, M. A. Verschuuren, K. Guo, A. F. Koenderink, J. G. Rivas, and S. R.-K. Rodriguez, "Directional sideward emission from luminescent plasmonic nanostructures," Opt. Express 24, A388 (2016).

${ }^{22}$ V. Mikhailov, G. A. Wurtz, J. Elliott, P. Bayvel, and A. V. Zayats, "Dispersing light with surface plasmon polaritonic crystals," Phys. Rev. Lett. 99, 083901 (2007).
${ }^{23}$ H. Gao, J. K. Hyun, M. H. Lee, J.-C. Yang, L. J. Lauhon, and T. W. Odom, "Broadband plasmonic microlenses based on patches of nanoholes," Nano Lett. 10, 4111-4116 (2010).

${ }^{24}$ F. M. Huang, T. S. Kao, V. A. Fedotov, Y. Chen, and N. I. Zheludev, "Nanohole array as a lens," Nano Lett. 8, 2469-2472 (2008).

${ }^{25}$ F. van Beijnum, P. J. van Veldhoven, E. J. Geluk, M. J. A. de Dood, G. W t Hooft, and M. P. van Exter, "Surface plasmon lasing observed in metal hole arrays," Phys. Rev. Lett. 110, 206802 (2013).

${ }^{26}$ A. H. Schokker and A. F. Koenderink, "Lasing at the band edges of plasmonic lattices," Phys. Rev. B 90, 155452 (2014).

${ }^{27}$ T. W. Ebbesen, H. J. Lezec, H. F. Ghaemi, T. Thio, and P. A. Wolff, "Extraordinary optical transmission through sub-wavelength hole arrays," Nature 391, 667-669 (1998)

${ }^{28}$ T. Thio, H. F. Ghaemi, H. J. Lezec, P. A. Wolff, and T. W. Ebbesen, "Surface-plasmon-enhanced transmission through hole arrays in Cr films," J. Opt. Soc. Am. B 16, 1743-1748 (1999).

${ }^{29}$ Q. Yu, P. Guan, D. Qin, G. Golden, and P. M. Wallace, "Inverted sizedependence of surface-enhanced Raman scattering on gold nanohole and nanodisk arrays," Nano Lett. 8, 1923-1928 (2008).

${ }^{30}$ M. E. Stewart, C. R. Anderton, L. B. Thompson, J. Maria, S. K. Gray, J. A. Rogers, and R. G. Nuzzo, "Nanostructured plasmonic sensors," Chem. Rev. 108, 494-521 (2008)

${ }^{31}$ C. Valsecchi and A. G. Brolo, "Periodic metallic nanostructures as plasmonic chemical sensors," Langmuir 29, 5638-5649 (2013).

${ }^{32}$ M.-C. Estevez, M. A. Otte, B. Sepulveda, and L. M. Lechuga, "Trends and challenges of refractometric nanoplasmonic biosensors: A review," Anal. Chim. Acta 806, 55-73 (2014).

${ }^{33} \mathrm{~J}$. Li, J. Ye, C. Chen, L. Hermans, N. Verellen, J. Ryken, H. Jans, W. Van Roy, V. V. Moshchalkov, L. Lagae, and P. Van Dorpe, "Biosensing using diffractively coupled plasmonic crystals: The figure of merit revisited," Adv. Opt. Mater. 3, 176-181 (2015).

${ }^{34} \mathrm{P}$. Singh, "SPR biosensors: Historical perspectives and current challenges," Sens. Actuators, B 229, 110-130 (2016).

${ }^{35}$ B. Spackova, P. Wrobel, M. Bockova, and J. Homola, "Optical biosensors based on plasmonic nanostructures: A review," Proc. IEEE 104, 2380-2408 (2016).

${ }^{36}$ W. L. Barnes, T. W. Preist, S. C. Kitson, J. R. Sambles, N. P. K. Cotter, and D. J. Nash, "Photonic gaps in the dispersion of surface plasmons on gratings," Phys. Rev. B 51, 11164-11167 (1995).

${ }^{37}$ S. C. Kitson, W. L. Barnes, and J. R. Sambles, "Surface-plasmon energy gaps and photoluminescence," Phys. Rev. B 52, 11441-11445 (1995).

${ }^{38}$ S. C. Kitson, W. L. Barnes, and J. R. Sambles, "Full photonic band gap for surface modes in the visible," Phys. Rev. Lett. 77, 2670-2673 (1996).

${ }^{39}$ E. Devaux, T. W. Ebbesen, J.-C. Weeber, and A. Dereux, "Launching and decoupling surface plasmons via micro-gratings," Appl. Phys. Lett. 83, 4936-4938 (2003).

${ }^{40}$ A. Drezet, A. Hohenau, A. L. Stepanov, H. Ditlbacher, B. Steinberger, F. R. Aussenegg, A. Leitner, and J. R. Krenn, "Surface plasmon polariton Mach-Zehnder interferometer and oscillation fringes," Plasmonics 1, 141-145 (2006).

${ }^{41}$ A. Drezet, D. Koller, A. Hohenau, A. Leitner, F. R. Aussenegg, and J. R. Krenn, "Plasmonic crystal demultiplexer and multiports," Nano Lett. 7, 1697-1700 (2007).

${ }^{42}$ B. Stein, J.-Y. Laluet, E. Devaux, C. Genet, and T. W. Ebbesen, "Surface plasmon mode steering and negative refraction," Phys. Rev. Lett. 105, 266804 (2010).

${ }^{43}$ B. Stein, E. Devaux, C. Genet, and T. W. Ebbesen, "Self-collimation of surface plasmon beams," Opt. Lett. 37, 1916-1918 (2012).

${ }^{44}$ D. Canneson, E. L. Moal, S. Cao, X. Quélin, H. Dallaporta, G. Dujardin, and E. Boer-Duchemin, "Surface plasmon polariton beams from an electrically excited plasmonic crystal,” Opt. Express 24, 26186-26200 (2016).

${ }^{45}$ A. Yang, Z. Li, M. P. Knudson, A. J. Hryn, W. Wang, K. Aydin, and T. W. Odom, "Unidirectional lasing from template-stripped two-dimensional plasmonic crystals," ACS Nano 9, 11582-11588 (2015).

${ }^{46}$ A. Abbas, M. J. Linman, and Q. Cheng, "New trends in instrumental design for surface plasmon resonance-based biosensors," Biosens. Bioelectron. 26, 1815-1824 (2011).

${ }^{47}$ M. Sarkar, M. Besbes, J. Moreau, J.-F. Bryche, A. Olivéro, G. Barbillon, A.-L. Coutrot, B. Bartenlian, and M. Canva, "Hybrid plasmonic mode by resonant coupling of localized plasmons to propagating plasmons in a Kretschmann configuration," ACS Photonics 2, 237-245 (2015).

${ }^{48} \mathrm{H}$. A. Atwater and A. Polman, "Plasmonics for improved photovoltaic devices," Nat. Mater. 9, 205-213 (2010). 
${ }^{49}$ D. Dominguez, N. Alharbi, M. Alhusain, A. A. Bernussi, and L. Grave de Peralta, "Fourier plane imaging microscopy," J. Appl. Phys. 116, 103102 (2014).

${ }^{50}$ D. B. Desai, M. M. S. Aldawsari, B. M. H. Alharbi, S. Sen, and L. Grave de Peralta, "Comprehensive study of unexpected microscope condensers formed in sample arrangements commonly used in optical microscopy," Appl. Opt. 54, 7781 (2015).

${ }^{51}$ D. B. Desai and L. Grave de Peralta, "Optical condensers formed in wetmounting setup," Appl. Opt. 54, 3580 (2015).

${ }^{52}$ D. Dominguez, M. Alhusain, N. Alharbi, A. Bernussi, and L. Grave de Peralta, "Fourier plane imaging microscopy for detection of plasmonic crystals with periods beyond the optical diffraction limit," Plasmonics 10, 1337-1344 (2015).

${ }^{53}$ J.-F. Bryche, R. Gillibert, G. Barbillon, M. Sarkar, A.-L. Coutrot, F. Hamouda, A. Aassime, J. Moreau, M. Lamy de la Chapelle, B. Bartenlian, and M. Canva, "Density effect of gold nanodisks on the SERS intensity for a highly sensitive detection of chemical molecules," J. Mater. Sci. 50, 6601-6607 (2015).

${ }^{54}$ J.-F. Bryche, R. Gillibert, G. Barbillon, P. Gogol, J. Moreau, M. Lamy de la Chapelle, B. Bartenlian, and M. Canva, "Plasmonic enhancement by a continuous gold underlayer: Application to SERS sensing," Plasmonics 11, 601-608 (2016).

${ }^{55}$ Y. Chu, E. Schonbrun, T. Yang, and K. B. Crozier, "Experimental observation of narrow surface plasmon resonances in gold nanoparticle arrays," Appl. Phys. Lett. 93, 181108 (2008).

${ }^{56}$ G. F. Iriarte, J. G. Rodriguez-Madrid, and F. Calle, "Fabrication of sub-100 nm IDT SAW devices on insulating, semiconducting and conductive substrates," J. Mater. Process. Technol. 212, 707-712 (2012).

${ }^{57}$ Y. Wang, M. Abb, S. A. Boden, J. Aizpurua, C. H. de Groot, and O. L. Muskens, "Ultrafast nonlinear control of progressively loaded, single plasmonic nanoantennas fabricated using helium ion milling," Nano Lett. 13, 5647-5653 (2013).

${ }^{58}$ S. Cao, E. Le Moal, F. Bigourdan, J.-P. Hugonin, J.-J. Greffet, A. Drezet, S. Huant, G. Dujardin, and E. Boer-Duchemin, "Revealing the spectral response of a plasmonic lens using low-energy electrons," Phys. Rev. B 96, 115419 (2017).

${ }^{59}$ J. Homola, S. S. Yee, and G. Gauglitz, "Surface plasmon resonance sensors: Review," Sens. Actuators, B 54, 3-15 (1999).

${ }^{60}$ R. M. A. Azzam, "Phase shifts that accompany total internal reflection at a dielectric - dielectric interface," J. Opt. Soc. Am. A 21, 1559-1563 (2004).
${ }^{61}$ R. M. A. Azzam, "Phase shifts in frustrated total internal reflection and optical tunneling by an embedded low-index thin film," J. Opt. Soc. Am. A 23, 960-965 (2006).

${ }^{62}$ L. Salomon, F. de Fornel, and P. M. Adam, "Analysis of the near field and the far field diffracted by a metallized grating at and beyond the plasmon resonance," J. Opt. Soc. Am. A 16, 2695-2704 (1999).

${ }^{63}$ A. Hohenau, J. R. Krenn, F. J. Garcia-Vidal, S. G. Rodrigo, L. MartinMoreno, J. Beermann, and S. I. Bozhevolnyi, "Spectroscopy and nonlinear microscopy of gold nanoparticle arrays on gold films," Phys. Rev. B 75, 085104 (2007).

${ }^{64}$ S. Lal, S. Link, and N. J. Halas, "Nano-optics from sensing to waveguiding," Nat. Photonics 1, 641-648 (2007).

${ }^{65}$ E. Yablonovitch, "Inhibited spontaneous emission in solid-state physics and electronics,” Phys. Rev. Lett. 58, 2059-2062 (1987).

${ }^{66}$ D. Brissinger, L. Salomon, and F. De Fornel, "Unguided plasmon-mode resonance in optically excited thin film: Exact modal description of Kretschmann-Raether experiment," J. Opt. Soc. Am. B 30, 333-337 (2013).

${ }^{67}$ C. Fallet, T. Novikova, M. Foldyna, S. Manhas, B. Haj Ibrahim, A. De Martino, C. Vannuffel, and C. Constancias, "Overlay measurements by Mueller polarimetry in back focal plane," J. Micro/Nanolithog. MEMS, MOEMS 10, 033017 (2011).

${ }^{68}$ S. Liu, W. Du, X. Chen, H. Jiang, and C. Zhang, "Mueller matrix imaging ellipsometry for nanostructure metrology," Opt. Express 23, 17316-17329 (2015).

${ }^{69}$ V. Ferreras Paz, S. Peterhnsel, K. Frenner, and W. Osten, "Solving the inverse grating problem by white light interference Fourier scatterometry," Light Sci. Appl. 1, e36 (2012).

${ }^{70}$ N. Kumar, P. Petrik, G. K. P. Ramanandan, O. E. Gawhary, S. Roy, S. F. Pereira, W. M. J. Coene, and H. P. Urbach, "Reconstruction of subwavelength features and nano-positioning of gratings using coherent Fourier scatterometry," Opt. Express 22, 24678-24688 (2014).

${ }^{71}$ H. F. Talbot, "LXXVI. Facts relating to optical science. No. IV," London Edinburgh Dublin Philos. Mag. J. Sci. 9, 401-407 (1836).

${ }^{72}$ L. Rayleigh, "XXV. On copying diffraction-gratings, and on some phenomena connected therewith," London Edinburgh Dublin Philos. Mag. J. Sci. 11, 196-205 (1881).

${ }^{73}$ W. Li, H. Li, B. Gao, and Y. Yu, "Investigation on the plasmon Talbot effect of finite-sized periodic arrays of metallic nanoapertures," Sci. Rep. 7, 45573 (2017)

${ }^{74}$ L. Zhu, R. Badugu, D. Zhang, R. Wang, E. Descrovi, and J. R. Lakowicz, "Radiative decay engineering 8: Coupled emission microscopy for lens-free high-throughput fluorescence detection," Anal. Biochem. 531, 20-36 (2017). 